\title{
Long-Term Follow Up of Patients with Mild-to-Moderate Alzheimer's Disease Treated with Bapineuzumab in a Phase III, Open-Label, Extension Study
}

\author{
Stephen P. Salloway ${ }^{\mathrm{a}, \#}$, Reisa Sperling ${ }^{\mathrm{b}, \#}$, Nick C. Fox ${ }^{\mathrm{c}}$, Marwan N. Sabbagh ${ }^{\mathrm{d}, 1}$, \\ Lawrence S. Honig ${ }^{\mathrm{e}}$, Anton P. Porsteinsson ${ }^{\mathrm{f}}$, Hany Rofael ${ }^{\mathrm{g}}$, Nzeera Ketterg, ${ }^{\mathrm{g}}$, Daniel Wang ${ }^{\mathrm{g}}$, \\ Enchi Liu ${ }^{\mathrm{g}, 4}$, Stephen Carr ${ }^{\mathrm{g}}$, Ronald S. Black ${ }^{\mathrm{h}, 3}$ and H. Robert Brashear ${ }^{\mathrm{i}, *}$ \\ ${ }^{a}$ Brown Medical School, Butler Hospital, Providence, RI, USA \\ ${ }^{\mathrm{b}}$ Center for Alzheimer Research and Treatment, Brigham and Women's Hospital, MA, USA \\ ${ }^{\mathrm{c}}$ Dementia Research Centre, University College London, Institute of Neurology, London, UK \\ ${ }^{\mathrm{d}}$ Barrow Neurological Institute, Phoenix, AZ, USA \\ ${ }^{\mathrm{e}}$ Columbia University, New York, NY, USA \\ ${ }^{\mathrm{f}}$ University of Rochester School of Medicine and Dentistry, Rochester, NY, USA

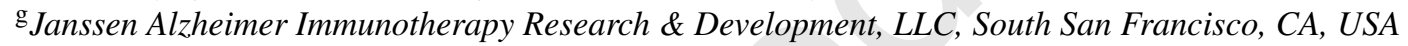 \\ ${ }^{\mathrm{h}}$ Pfizer Inc, Collegeville, PA, USA \\ ${ }^{\mathrm{i} J a n s s e n}$ Research \& Development LLC, Pennington, NJ, USA
}

Handling Associate Editor: Katherine Bangen

Accepted 7 May 2018

\begin{abstract}
. efficacy of bapineuzumab.

\footnotetext{
${ }^{1}$ Present address: Cleveland Clinic Lou Ruvo Center for Brain Health888 W Bonneville Ave., Las Vegas, NV, USA.

${ }^{2}$ Present address: 141 Leland Avenue, Menlo Park, CA, USA.

${ }^{3}$ Present address: Prothena Biosciences Inc., South San Francisco, CA, USA.

${ }^{4}$ Present address: 1625 Paper Mill Rd., Meadowbrook PA, USA.
}

Background: A 3-year extension of two Phase III parent studies of intravenous (IV) bapineuzumab in patients with mildto-moderate Alzheimer's disease dementia (apolipoprotein (APOE) $\varepsilon 4$ carriers and noncarriers) is summarized.

Objectives: The primary and secondary objectives were to evaluate the long-term safety, tolerability, and maintenance of

Methods: A multicenter study in patients who had participated in double-blind placebo-controlled parent studies. Patients enrolled in the extension study were assigned to receive IV infusions of bapineuzumab $(0.5 \mathrm{or} 1.0 \mathrm{mg} / \mathrm{kg})$ every $13 \mathrm{weeks}$ until termination but were blinded to whether they had received bapineuzumab or placebo in the parent studies.

Results: A total of 1,462 (688 were $A P O E \varepsilon 4$ carriers and 774 were noncarriers) patients were enrolled. Extension-onset adverse events occurred in $>81 \%$ of the patients in each dose group. Fall, urinary tract infection, agitation, and ARIA-E occurred in $\geq 10 \%$ of participants. The incidence proportion of ARIA-E was higher among carriers and noncarriers who received bapineuzumab for the first time in the extension study $(11.8 \%$ and $5.4 \%$, respectively) versus those who were 
previously exposed in the parent studies (5.1\% and 1.3\%, respectively). After 6 to 12 months exposure to bapineuzumab IV in the extension study, similar deterioration of cognition and function occurred with no significant differences between the dose groups.

Conclusions: Infusion of bapineuzumab 0.5 or $1.0 \mathrm{mg} / \mathrm{kg}$ every 13 weeks for up to 3 years was generally well tolerated, with a safety and tolerability profile similar to that in previous studies.

Keywords: Alzheimer's disease, amyloid-related imaging abnormality with edema/effusions, bapineuzumab, long-term safety

\section{INTRODUCTION}

Bapineuzumab is a recombinant humanized monoclonal antibody that targets the $\mathrm{N}$-terminus of the human amyloid- $\beta(A \beta)$ protein and was designed to lower cerebral $A \beta$ deposits in Alzheimer's disease (AD) $[1,2]$. Bapineuzumab was investigated as a disease-modifying therapy for treatment of $\mathrm{AD}$ in 15 clinical studies conducted over several years (2006-2012). The Phase III program consisted of four similarly designed studies conducted in parallel. Studies 301 and 302 (NCT00575055 and NCT00574132, respectively) were conducted primarily in the United States, and global Studies 3000 and 3001 (NCT00667810 and NCT00676143) were conducted at sites in more than 26 countries worldwide $[3,4]$.

No clinical benefit was observed in either Study 301 or 302; neither showed significant differences in clinical efficacy between bapineuzumab at these dosages and placebo groups [3,5]. Lack of efficacy on clinical endpoints was later confirmed in the global Studies 3000 and 3001, and the extension phases of both of these latter studies were terminated early [4]. Bapineuzumab clinical development was discontinued in December 2012.

Biomarkers tested in subsets of participants in the bapineuzumab studies included cerebrospinal fluid (CSF) phosphorylated-tau (p-tau), total tau (T-tau), CSF $A \beta_{1-42}$, and amyloid positron emission tomography (PET). CSF p-tau and T-tau are elevated in patients with $\mathrm{AD}$, reflecting $\mathrm{AD}$-related neurodegeneration, while CSF $A \beta_{1-42}$, a marker of cerebral amyloid accumulation, is reduced [6, 7]. Although no clinical benefit was demonstrated, treatment with bapineuzumab over 18 months reduced the concentration of p-tau in CSF relative to placebo in APOE $\varepsilon 4$ carriers, and in the $1.0 \mathrm{mg} / \mathrm{kg}$ dose level in noncarriers but not in the $0.5 \mathrm{mg} / \mathrm{kg}$ dose level or in pooled analyses of doses in noncarriers [3, 8]. Brain amyloid burden assessed by ${ }^{11} \mathrm{C}$-Pittsburgh compound B-PET imaging of a subset of participants in phase
III trials was significantly reduced in carriers and in pooled analyses of both carriers and noncarriers versus placebo but not in the noncarriers separately [9]. Findings from phase II PET studies also had shown reduction of the mean ${ }^{11} \mathrm{C}$-PiB retention ratio from baseline to week 78 for the bapineuzumab group relative to the placebo group, indicative of some target engagement by bapineuzumab on amyloid plaques [10].

In bapineuzumab Phase II studies, parenchymal and sulcal hyperintensities were seen on fluid attenuated inversion recovery (FLAIR) sequence of magnetic resonance imaging (MRI) scans consistent with edema or effusion, termed ARIA-E (amyloidrelated imaging abnormalities with edema/effusion). A greater incidence of ARIA-E was reported with increasing bapineuzumab dose and with copy number of Apolipoprotein E (APOE) $\varepsilon 4$ allele [11]. The bapineuzumab $2.0 \mathrm{mg} / \mathrm{kg}$ dose was discontinued early (approximately 14 months after trial initiation) in the APOE \&4 noncarrier trials 301 and 3000 due to a higher rate of clinically symptomatic ARIA-E (7.8\% versus $1.5 \%$ in 0.5 and $1.0 \mathrm{mg} / \mathrm{kg}$ dose groups respectively in 301 trial), and patients who were initially randomized to $2.0 \mathrm{mg} / \mathrm{kg}$ were reassigned to the $1.0 \mathrm{mg} / \mathrm{kg}$ group.

ARIA-E was confirmed as the most notable adverse event (AE) in the phase III studies. The Final Read of MRI brain scans assessed after participants completed Study 301 or Study 302 showed that treatment-emergent ARIA-E occurred in $15.8 \%$ of bapineuzumab and $0.8 \%$ of placebo-treated patients respectively. ARIA-E occurred in 5.6\%, 13.4\%, and $19.9 \%$ of $A P O E \& 4$ noncarriers in $0.5,1.0$, and $2.0 \mathrm{mg} / \mathrm{kg}$ groups, respectively, and in $21.2 \%$ of carriers in the $0.5 \mathrm{mg} / \mathrm{kg}$ group [12]. In the global studies, incidences of ARIA-E in noncarriers were $4.9 \%$ and $11.8 \%$ in bapineuzumab $0.5 \mathrm{mg} / \mathrm{kg}$ and $1.0 \mathrm{mg} / \mathrm{kg}$ groups respectively and $0.6 \%$ in the placebo group, and for $A P O E \varepsilon 4$ carriers ARIA-E occurred in $16.7 \%$ of the $0.5 \mathrm{mg} / \mathrm{kg}$ group and $2.1 \%$ of the placebo group [4]. 
Long term safety and tolerability of bapineuzumab in patients with $\mathrm{AD}$ were reported in two other extension studies that were terminated early due to lack of efficacy in phase III trials, Study 3002 with noncarriers (NCT00996918) and Study 3003 with carriers (NCT00998764) were continued from their two global phase III parent studies [13]. Patients who were receiving bapineuzumab continued at the same dose and patients receiving placebo began bapineuzumab in the extension studies. ARIA-E identified during both extension studies was the main bapineuzumabassociated AE, overall occurring in approximately $11 \%$ of placebo $\rightarrow$ bapineuzumab (pbo $\rightarrow$ bapi) and $4 \%$ of bapineuzumab $\rightarrow$ bapineuzumab (bapi $\rightarrow$ bapi) groups. Exploratory analysis showed that clinical efficacy was not significantly different between groups in either study [13].

Here we report the findings from the open-label extension study conducted in patients with $\mathrm{AD}$ who participated in the parent phase III Studies 301 and 302 that evaluated $A P O E \varepsilon 4$ allele noncarriers and carriers in the same bapineuzumab dose groups as separate study cohorts. In this study, we examined the longer-term effects of bapineuzumab on the incidence and severity of clinically relevant AEs. Cognitive and functional efficacy endpoint changes over time (from the parent study and this study baselines), and changes from extension study baseline in AD-related health outcome measures and clinical biomarkers were also assessed.

\section{METHODS}

\section{Overview of study design}

This was a multicenter, long-term safety and tolerability open-label phase III extension study (Study 351, NCT00937352) of intravenous (IV) bapineuzumab in patients with mild-to-moderate $\mathrm{AD}$ who participated in Study 301 (APOE $\varepsilon 4$ noncarriers) or in Study 302 (APOE \&4 carriers). The parent studies were two multicenter, randomized, double-blind, placebo-controlled studies that were previously published [3]. Each study consisted of a 6 -week screening period, 65 weeks of dosing during which bapineuzumab or placebo was infused intravenously every 13 weeks (day 1, weeks 13, 26, 39, 52 , and 65), and a follow-up period of 13 weeks.

Study 351 was conducted at 184 sites in the US (171), Canada (12), and Germany (1) from October 2009 to September 2012. Approximately 1000 patients from Study 301 and 700 from Study
302 were enrolled in this extension study. Patients without ARIA-E in Study 301 who had received bapineuzumab continued to receive treatment with bapineuzumab IV once every 13 weeks at the same assigned dose level (either 0.5 or $1.0 \mathrm{mg} / \mathrm{kg}$ ). Patients who had received placebo in the parent study, were randomized to receive either bapineuzumab $0.5 \mathrm{mg} / \mathrm{kg}$ or $1.0 \mathrm{mg} / \mathrm{kg}$ at a ratio of $1: 1$ without stratification. Patients who originally received the discontinued $2.0 \mathrm{mg} / \mathrm{kg}$ dose in the Study 301 were reassigned to $1.0 \mathrm{mg} / \mathrm{kg}$ during that study and continued to receive the $1.0 \mathrm{mg} / \mathrm{kg}$ dose in this extension study.

For this extension study, $A P O E \varepsilon 4$ carriers (from Study 302) were assigned to bapineuzumab $0.5 \mathrm{mg} / \mathrm{kg}$ whether they had received bapineuzumab or placebo. Patients who were on active treatment in Study 301 and had experienced ARIA-E prior to their enrollment in Study 351 were assigned to bapineuzumab $0.5 \mathrm{mg} / \mathrm{kg}$ dose group. As per protocol, a redosing scheme was used for patients who were detected with ARIA-E at the last visit (Visit 15/Week 78) of either parent study. Accordingly, those patients who had previously received bapineuzumab $0.5 \mathrm{mg} / \mathrm{kg}$ or placebo prior to their enrollment in Study 351 were assigned to a starting dose of $0.15 \mathrm{mg} / \mathrm{kg}$, and if they had received $1.0 \mathrm{mg} / \mathrm{kg}$, they were assigned to a starting dose of $0.5 \mathrm{mg} / \mathrm{kg}$ in the extension study. Following resolution of ARIA-E MRI findings and any clinical findings, the bapineuzumab $0.15 \mathrm{mg} / \mathrm{kg}$ starting dose was increased to $0.5 \mathrm{mg} / \mathrm{kg}$ at the next scheduled dose and was maintained at the same level for all subsequent infusions.

This study design resulted in an early-start bapineuzumab treatment group that included all patients who had received active treatment in parent Studies 301 or 302 and continued after enrollment in Study 351 , and a delayed-start treatment group of those who had received placebo prior to Study 351. Bapineuzumab dose level assignment for noncarriers and assignment to treatment in parent studies remained blinded to patients, sponsor staff and medical site staff.

Study 351 was approved by each participating local site institutional review board or independent ethics committee and written informed consent was obtained from each patient (or legally authorized representative). The study was conducted in accordance with the ethical principles that have their origin in the Declaration of Helsinki and that are consistent with International Conference on Harmonization for Good 
Clinical Practices guidelines and applicable local and regulatory requirements.

\section{Population}

Patients were 50 to 88 years of age at the time of trial entry and met the inclusion criteria for the parent Studies 301 and 302 [3]. Those eligible to participate in this study had to have completed all 6 study drug infusions as per parent protocol, unless they were required to temporarily suspend treatment, and met the following criteria: continued with the required clinic visits, were eligible to resume treatment, completed through to last Visit 15/Week 78 of parent studies, and had a brain MRI scan at Visit 14/Week 71 of either parent study. Also, they had to continue to live at home or in a community dwelling with an appropriate caregiver and be available for all onsite evaluations for the duration of the study. Patients who consented to participate in PET, volumetric MRI (vMRI), or CSF p-tau substudy of Study 351 must have completed parent 301/302Visit 14/Week $71 \mathrm{PiB}$ PET, vMRI scan or a lumbar puncture procedure.

Patients were excluded from being enrolled into the extension study if they had any new medical contraindication or clinically significant abnormality on physical, neurological, laboratory, vital signs or electrocardiogram (ECG) examination (e.g., atrial fibrillation) that in the investigator's opinion precluded continued or initiation of treatment with bapineuzumab or participation and evaluation of response in the study. Patients were also excluded if a screening visit brain MRI scan Week 71 of the parent study indicated any significant abnormality such as evidence of prior hemorrhage $>1 \mathrm{~cm}^{3}$, more than 4 microhemorrhages $(<10 \mathrm{~mm})$, or evidence of a single prior infarct $>1 \mathrm{~cm}^{3}$, cerebral contusion, encephalomalacia, aneurysms, vascular malformations, subdural hematoma, or space occupying lesions (e.g., brain tumor such as meningioma). Per protocol, other exclusion criteria were neurologic disease other than $\mathrm{AD}$, major psychiatric disorder, history of stroke or seizures; current use of anticoagulants, use of antiplatelet therapy for stroke prophylaxis (but allowed for other indications), and treatment with cognitive enhancers other than stable doses of acetylcholinesterase inhibitors or memantine.

\section{Objectives}

The primary objectives were to evaluate the longterm safety and tolerability of bapineuzumab IV in patients with $\mathrm{AD}$ as assessed by the incidence and severity of treatment-emergent adverse events (TEAEs) and clinically important changes in vital signs, clinical laboratory tests, ECGs parameters, brain MRI scans, physical and neurological examinations, infusion site assessments and suicidality assessments.

Secondary efficacy objectives determined if efficacy persisted after 6 months of bapineuzumab IV treatment by measuring the changes from parent study baseline and extension study baseline in the Alzheimer's Disease Assessment Scale - Cognitive subscale (ADAS-Cog/11) and the Disability Assessment Scale for Dementia (DAD). Exploratory objectives evaluated the effect of long-term bapineuzumab IV treatment on other clinical biomarker, and health outcome measures.

\section{Schedule of events}

Baseline and screening procedures for participants who enrolled in Study 351 (Pre-Day 1) were performed at or before Visit 15/Week 78 of Study 301 or 302 in accordance with inclusion/exclusion eligibility criteria. Bapineuzumab infusions were administered on Day 1, and on Weeks 13, 26, 39, 52, 65, and every 13 weeks until end of study or early termination which was scheduled within 60 days post last dose ( \pm 14 days). Patients received their first bapineuzumab infusion no later than 19 weeks post last infusion (Visit 13/Week 65) in either Study 301 or 302. Patients whose ARIA-E was confirmed at Visit 14/Week 71 of Study 301 or 302 and approved by medical monitor to continue into Study 351, received their first infusion in the extension study no earlier than 26 weeks after the last infusion in either parent study to allow for resolution of ARIA radiographically.

Cognitive, functional and health outcome assessments were assessed at approximately 3-month intervals for the first year, then at 6-month intervals (Mini-Mental State Examination [MMSE], Dependence Scale [DS], Abbreviated Resource Utilization in Dementia [Abbr.RUD Lite v2.4], Health Utilities Index [HUI], and Neuropsychiatric Inventory [NPI]) and 12-month intervals (ADAS-Cog/11, DAD) until the end of study or early termination. Safety MRI scans were performed at baseline, at week 6 , and at approximately 3-month intervals in the first year (6 weeks after each infusion) then at 6-month intervals until end of study or early termination. PiB PET, vMRI, and CSF biomarker measurements were 
made at week 65 , then yearly until end of study or early termination visit (60 days after last infusion \pm 14 days)

\section{Study procedures}

\section{Safety assessments}

A TEAE was an AE that started during or after the first infusion of study drug (in Study 301 or 302) and prior to or on the date of last dose in the extension study +137 days. An AE with an onset during the extension study was called "extension-onset AE". Incidence and severity of TEAEs whether or not related to the study drug were monitored throughout the study and were solicited after ARIA-E was detected.

During the study, brain MRI scans were reviewed by local radiologists associated with each clinical site and by a central radiologist (Central Read) (BioClinica Inc., formerly Synarc, Newtown PA) for real-time safety monitoring and assessment of amyloid-related imaging abnormalities consisting of ARIA-E, ARIA-H (hemosiderin deposits), and intracerebral hemorrhages. Both local and central interpretation of the acquired MRI sequences (or the local report alone if the Central Read had not been received) were reviewed for clinical significance prior to the patient's next infusion visit.

In the blinded parent studies, a separate systematic, sequential locked retrospective review of all MRI scans by a pair of independent central neuroradiologists, called the MRI Final Read was instituted following the completion of patient's participation in either Study 301 and 302 [12]. A Final Read of all MRIs was not conducted in this extension study partly because ascertainment of ARIA-E cases was expected to improve due to increased familiarity of local and central readers and MRI procedures had evolved over the course of the study in accordance with sequential protocol amendments.

\section{Efficacy and biomarker assessments}

Longitudinal efficacy endpoints relative to the baseline in parent Study 301 or 302 (Visit 2/Pre-Day 1 ), and relative to the baseline in Study 351 (Visit 15/Week 78 of Study 301 or 302) were evaluated for ADAS-Cog/11, DAD, and MMSE. Change in health outcome measures were also evaluated: NPI assessed 12 neuropsychiatric disturbances common in dementia patients, DS a 13-item questionnaire administered to the caregiver assessed the required amount of assistance needed by the $\mathrm{AD}$ patient,
Abbr. RUD-Lite v.2.4 instrument administered to the caregiver/informant gathered information on medical support utilization and other services provided to the patient, and HUI questionnaire administered to the caregiver used to obtain health status information about the patient.

Effects of bapineuzumab on disease biomarkers were assessed in subsets of enrolled participants in the parent studies who agreed to participate in optional substudies. Brain amyloid burden was determined using ${ }^{11} \mathrm{C}-\mathrm{PiB}$ PET scan standardized uptake value ratio (SUVr) averaged from five cortical regions of interest (frontal, temporal, or parietal cortices, anterior and posterior cingulates, and cerebellum) with cerebellar gray matter as the reference region. Changes in CSF levels of $A \beta$, T-tau, p-tau, and in whole brain volume (WBV) and brain boundary shift integral (BBSI), ventricular volume (VV), and ventricular boundary shift integral (VBSI), and hippocampal boundary shift integral (HBSI) measured by volumetric brain MRI (vMRI).

\section{Study data analysis}

Sample size was the number of participants that completed Study 301 and 302, were eligible and were enrolled in the extension study. Due to decision to stop ongoing bapineuzumab IV studies for lack of efficacy, results from Study 351 were available after the first data-base lock in April 2012 and then finalized after analysis of final data base lock 7 months later. Safety analysis population included all patients who consented to participate, received any study drug in the extension study. Analyses were reported separately for carriers and noncarriers. Parent study modified intent-to-treat (mITT) analysis population included all randomized patients who received at least 1 infusion or portion of an infusion of study drug and who had a baseline and at least 1 postbaseline assessment of the ADAS-Cog/11 total score and DAD total score in Study 301 or 302 . This was the analysis population for Study 351 that supported the secondary efficacy objective. Biomarker analysis population included all randomized participants who had enrolled and received at least 1 or portion of an infusion of study drug. All enrolled participants in the $\mathrm{C}^{11}$-PiB PET assessment substudy were selected to have $\mathrm{SUVr} \geq 1.35$ at baseline.

All analyses were summarized by cohorts defined by patient's treatment in the parent Study 301 or 302 and the treatment in Study 351. Per protocol, separate and pooled bapineuzumab dose groups were defined. The pooled groups combined 0.5 and $1.0 \mathrm{mg} / \mathrm{kg}$ 


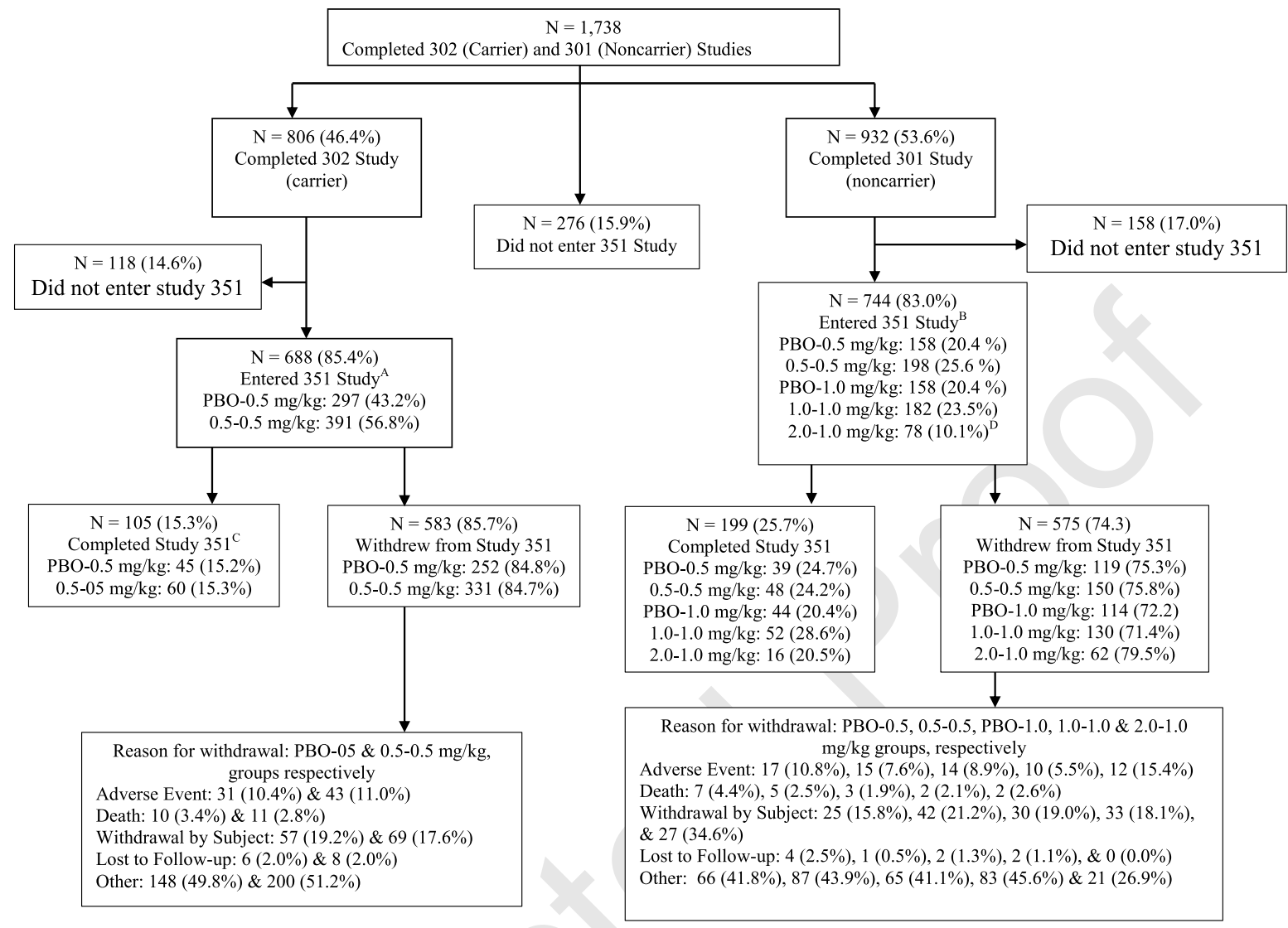

Fig. 1. Participant disposition for Study 351. ${ }^{\mathrm{A}}$ Treatment arm Study $302-351{ }^{\mathrm{B}}$ Treatment arm Study $301-351 .{ }^{\mathrm{C}}$ Completed $=$ continued in the study until it was terminated by the Sponsor on $21 \mathrm{Sept} .2012 .{ }^{\mathrm{D}}$ Bapineuzumab $2.0 \mathrm{mg} / \mathrm{kg}$ group discontinued during the course of Study 301.

dose groups and were termed early start, or previously exposed, bapi $\rightarrow$ bapi and delayed start or newly exposed pbo $\rightarrow$ bapi dose groups. Analyses of treatment groups were performed for combined treatment groups, and separately for carriers and non-carriers. In Study 301, patients who were initially assigned to the bapineuzumab $2.0 \mathrm{mg} / \mathrm{kg}$ treatment group and subsequently received bapineuzumab $1.0 \mathrm{mg} / \mathrm{kg}$ were analyzed separately. These participants were also analyzed separately in Study 351, but not included in pooled early start group or in any statistical analyses.

Mean change from baseline over time was presented in two ways: 1) using the parent baseline (last value measured prior to the first dose of study treatment in the Study 301 or 302), and reporting changes over the time periods of all three Studies $(301,302$, and 351), and 2) using the baseline from Study 351 (i.e., last value measured prior to first dose of bapineuzumab in Study 351). Changes from extension baseline over time were used for safety evaluations. Change from parent baseline to extension Week 65 was used for analysis of secondary efficacy endpoints of ADAS-Cog/11 total score and DAD total score (parent study mITT analysis population) and exploratory clinical and biomarker endpoints.

Primary efficacy analysis evaluated longitudinal early start versus delayed start treatment differences for maintenance of bapineuzumab clinical efficacy after 6 months of treatment in the extension study. A mixed model for repeated measures (MMRM) was used to analyze the change in ADAS-Cog/11 total score and DAD total score from the parent baseline using PROC MIXED in SAS Statistical software, version 9.2 (SAS institute, Cary NC). Treatment differences (bapi $\rightarrow$ bapi minus pbo $\rightarrow$ bapi) were estimated using least-square means with factor levels weighted according to overall baseline sample proportions. MMRM Model 1 was used for analyses based on parent Study 301 alone (APOE $\varepsilon 4$ noncarriers), whereas MMRM Model 2 was used for analyses of parent Study 302 alone. Model 2 includes number of $A P O E \varepsilon 4$ alleles (1 or 2) as a factor. The same 
MMRM models were used for analyses of PiB PET, CSF, and vMRI data.

\section{RESULTS}

\section{Patient disposition and exposure}

Patient disposition in the extension study is shown in Fig. 1. A total of 2,452 participants were randomized in the 2 parent studies. Of these, 1,738 participants completed the parent studies, 1462 were subsequently enrolled in the extension study, received bapineuzumab and included in the safety analysis population, of whom $688(47.1 \%)$ were $A P O E \varepsilon 4$ carriers and $774(52.9 \%)$ were noncarriers. A total of 2,204 participants were in the parent study mITT analysis population which was the analysis population for the primary efficacy analysis of Study 351 (in support of secondary efficacy objectives). Of these, 1090 were carriers with 432 in pbo $\rightarrow$ bapi $0.5 \mathrm{mg} / \mathrm{kg}$, and 658 in bapi $0.5 \mathrm{mg} / \mathrm{kg} \rightarrow$ bapi $0.5 \mathrm{mg} / \mathrm{kg}$ dose groups; and 1114 were noncarriers with 493 in pooled pbo $\rightarrow$ bapi 0.5 , and $1.0 \mathrm{mg} / \mathrm{kg}$ dose groups and 621 in pooled bapi $\rightarrow$ bapi $0.5,1.0 \mathrm{mg} / \mathrm{kg}$ dose groups. There were 78 noncarriers initially assigned to the $2.0 \mathrm{mg} / \mathrm{kg}$ in Study 301, reassigned to the $1.0 \mathrm{mg} / \mathrm{kg}$ dose in Study 301 who continued the $1.0 \mathrm{mg} / \mathrm{kg}$ dose in Study 351 and were not included in the mITT analysis population but were included in the safety analysis population. Percentages of carriers and noncarriers who continued to participate in the extension study until it was terminated by the sponsor were $15.3 \%$ (105/688) and 25.7\% (199/774) respectively. The most common reasons for withdrawal among both carriers and noncarriers were "withdrawal by subject" (carriers: 21.6\%, noncarriers: $27.3 \%$ ) and "withdrawal due to adverse event" which accounted for $12.7 \%$ among carriers and $11.8 \%$ among noncarriers. Termination resulting from "physician decision" and "sponsor decision" was $48.9 \%$ for carriers, and $51.1 \%$ for noncarriers, the majority of which resulted from study termination due to sponsor decision.

The parent studies were 1.5 years in duration. The mean (SD) person-years of exposure to bapineuzumab in the parent and extension studies for the pbo $\rightarrow$ bapi dose groups was $1.41(0.828)$ years for carriers and $1.31(0.748)$ years for noncarriers. In the bapi $\rightarrow$ bapi dose groups, the mean extent of exposure among carriers and noncarriers was 3.06 (0.809) years and $2.88(0.721)$ years, respectively (Table 1).

\section{Demographics and baseline characteristics}

Parent study baseline demographic characteristics were comparable for carriers and noncarriers and, within each cohort, for the pbo $\rightarrow$ bapi and bapi $\rightarrow$ bapi dose groups (Table 1). Among the carriers, the mean age was 71.4 years in the pbo $\rightarrow$ bapi group and 70.9 years in the bapi $\rightarrow$ bapi group. The majority was white $(97.0 \%$ and $95.1 \%$, respectively) and more than half were female $(59.3 \%$ and $54.2 \%$, respectively). Among noncarriers, the mean age was 70.5 years in the pbo $\rightarrow$ bapi group and 71.9 years in the bapi $\rightarrow$ bapi group. Most were white $(94.6 \%$ and $93.9 \%$, respectively) and approximately half were female (50.9\% and $52.1 \%$, respectively).

Baseline characteristics were similar between the parent studies and the extension study. Like the parent study baseline, the percentage of participants with mild $\mathrm{AD}$ at the extension study baseline remained higher among the noncarriers $(41.8 \%$ in pbo $\rightarrow$ bapi; $44.4 \%$ in bapi $\rightarrow$ bapi) than the carriers $(32.5 \%$ and $35.4 \%$, respectively) (Table 1). Among APOE $\varepsilon 4$ noncarriers, $64.3 \%$ in the pbo $\rightarrow$ bapi group and $63.5 \%$ in the bapi $\rightarrow$ bapi group had mild AD (MMSE total score $\geq 21$ ) at parent Study 301 baseline. MMSE values ranged from 16 to 26 . Among $A P O E \varepsilon 4$ carriers, $52.1 \%$ in the pbo $\rightarrow$ bapi group and $56.6 \%$ in the bapi $\rightarrow$ bapi group had mild AD at parent Study 302 baseline, MMSE values ranged from 14 to 27 . For both carriers and noncarriers, MMSE values at extension study baseline ranged from 30 to as low as 0 , indicating that some patients had marked decline in dementia.

\section{Safety assessment}

An overview of TEAEs in both carriers and noncarriers, is shown in Table 2. In carriers, extension-onset AEs occurred in $83.5 \%$ of the patients in the pbo $\rightarrow$ bapi group and $87.2 \%$ of those in the bapi $\rightarrow$ bapi group, and the AEs were judged by investigators to be related to study drug in $26.6 \%$ and $18.2 \%$ of groups, respectively. In noncarriers, the incidence of corresponding AEs was $82.3 \%$ in pbo $\rightarrow$ bapi group and $81.1 \%$ in bapi $\rightarrow$ bapi group and study drug related AEs was in $16.8 \%$ and $12.9 \%$ of cohorts, respectively. Within each treatment dose group, the overall extension-onset $\mathrm{AE}$ profile was comparable for the pbo $\rightarrow$ bapi and bapi $\rightarrow$ bapi groups, although the percentages of carriers with AEs related to study drug and severe AEs (SAEs) were higher in the pbo $\rightarrow$ bapi group (26.6\% and $30.0 \%$, 
Table 1

Demographics and baseline characteristics of patients enrolled in Study 351 at parent study baseline (Safety Population)

\begin{tabular}{|c|c|c|c|c|c|c|c|c|c|}
\hline \multirow[t]{3}{*}{ Baseline Characteristic } & \multirow{2}{*}{\multicolumn{2}{|c|}{$\begin{array}{c}\text { APOE } \varepsilon 4 \text { Carriers } \\
\text { Study } 302 \rightarrow 351\end{array}$}} & \multicolumn{7}{|c|}{$A P O E \varepsilon 4$ Noncarriers Study $301 \rightarrow 351$} \\
\hline & & & \multirow[b]{2}{*}{$\begin{array}{c}\mathrm{pbo} \rightarrow \text { Bapi } \\
0.5 \mathrm{mg} / \mathrm{kg} \\
(\mathrm{N}=158) \\
\end{array}$} & \multicolumn{3}{|c|}{ Individual Doses } & \multirow[b]{2}{*}{$\begin{array}{c}\text { Bapi } 2.0(1.0) \\
\rightarrow 1.0 \mathrm{mg} / \mathrm{kg} \\
(\mathrm{N}=78)\end{array}$} & \multicolumn{2}{|c|}{ Pooled Doses } \\
\hline & $\begin{array}{c}\text { pbo } \rightarrow \text { Bapi } \\
0.5 \mathrm{mg} / \mathrm{kg} \\
(\mathrm{N}=297) \\
\end{array}$ & $\begin{array}{c}\text { Bapi } \\
0.5 \rightarrow 0.5 \mathrm{mg} / \mathrm{kg} \\
(\mathrm{N}=391)\end{array}$ & & $\begin{array}{c}\text { Bapi } \\
0.5 \rightarrow 0.5 \mathrm{mg} / \mathrm{kg} \\
(\mathrm{N}=198)\end{array}$ & $\begin{array}{c}\text { pbo } \rightarrow \text { Bapi } \\
1.0 \mathrm{mg} / \mathrm{kg} \\
(\mathrm{N}=158) \\
\end{array}$ & $\begin{array}{c}\text { Bapi } \\
\begin{array}{c}1.0 \rightarrow \\
\rightarrow\end{array} 1.0 \mathrm{mg} / \mathrm{kg} \\
(\mathrm{N}=182)\end{array}$ & & $\begin{array}{c}\text { pbo } \rightarrow \text { Bapi } \\
(\mathrm{N}=316)\end{array}$ & $\begin{array}{l}\text { Bapi } \rightarrow \text { Bapi } \\
(\mathrm{N}=380)\end{array}$ \\
\hline Age, y & $71.4 \pm 8.49$ & $70.9 \pm 8.15$ & $70.3 \pm 10.58$ & $72.3 \pm 9.11$ & $70.7 \pm 9.86$ & $71.4 \pm 9.48$ & $72.9 \pm 10.30$ & $70.5(10.21)$ & $71.9(9.29)$ \\
\hline \multicolumn{10}{|l|}{ Age Category, $n(\%)$} \\
\hline$<65 \mathrm{y}$ & 65 (21.9) & $86(22.0)$ & $53(33.5)$ & $42(21.2)$ & $46(29.1)$ & $48(26.4)$ & $20(25.6)$ & 99 (31.3) & $90(23.7)$ \\
\hline$\geq 65 \mathrm{y}$ & $232(78.1)$ & $305(78.0)$ & $105(66.5)$ & $156(78.8)$ & $112(970.9)$ & 134 (73.6) & $58(74.4)$ & $217(68.7)$ & $290(76.3)$ \\
\hline Female Sex, $n(\%)$ & $176(59.3)$ & $212(54.2)$ & $92(58.2)$ & $101(51.0)$ & $69(43.7)$ & $97(53.3)$ & $44(56.4)$ & $161(50.9)$ & $198(52.1)$ \\
\hline \multicolumn{10}{|l|}{ Race, $n(\%)$} \\
\hline Asian & $1(0.3)$ & $2(0.5)$ & $2(1.3)$ & $2(1.0)$ & $1(0.6)$ & $1(0.5)$ & $3(3.8)$ & $3(0.9)$ & $3(0.8)$ \\
\hline Black or African American & $4(1.3)$ & $14(3.6)$ & $7(4.4)$ & $5(2.5)$ & $1(0.6)$ & $8(4.4)$ & $1(1.3)$ & $8(2.5)$ & $13(3.4)$ \\
\hline White & $288(97.0)$ & $372(95.1)$ & $145(91.8)$ & $188(94.9)$ & $154(97.5)$ & $169(92.9)$ & 73 (93.6) & $299(94.6)$ & 357 (93.9) \\
\hline \multicolumn{10}{|c|}{ First Degree Relatives with AD, $n(\%)$} \\
\hline No & $132(44.4)$ & $180(46.0)$ & $88(55.7)$ & $132(66.7)$ & $86(54.4)$ & $110(60.4)$ & $38(48.7)$ & $174(55.1)$ & $242(63.7)$ \\
\hline Yes & $154(51.9)$ & $199(50.9)$ & $60(38.0)$ & $58(29.3)$ & $63(39.9)$ & $57(31.3)$ & $38(48.7)$ & $123(38.9)$ & $115(30.3)$ \\
\hline Years of Formal Education & $14.6 \pm 3.01$ & $14.6 \pm 2.83$ & $14.3 \pm 3.17$ & $14.4 \pm 3.09$ & $14.5 \pm 3.37$ & $14.5 \pm 3.32$ & $14.3 \pm 3.29$ & $14.4(3.27)$ & $14.5(3.20)$ \\
\hline $\begin{array}{l}\text { MMSE Total Score at Parent } \\
\text { Baseline, } \mathrm{N}^{*}\end{array}$ & 286 & 378 & 148 & 181 & 149 & 170 & 78 & 297 & 351 \\
\hline$<21$ (Mod. AD), $n(\%)$ & $137(47.9)$ & $164(43.4)$ & $51(34.5)$ & $70(38.7)$ & $55(36.9)$ & $58(34.1)$ & $30(38.5)$ & $106(35.7)$ & $128(36.5)$ \\
\hline$\geq 21$ (Mild AD), $n(\%)$ & $149(52.1)$ & $214(56.6)$ & $97(65.5)$ & $111(61.3)$ & $94(63.1)$ & $112(65.9)$ & $48(61.5)$ & $191(64.3)$ & $223(63.5)$ \\
\hline $\begin{array}{l}\text { MMSE Total Score at Extension } \\
\text { Baseline, } \mathrm{N}^{*}\end{array}$ & 286 & 378 & 148 & 181 & 149 & 170 & 78 & 297 & 351 \\
\hline$<21$ (Mod. AD), $n(\%)$ & $193(67.5)$ & $244(64.6)$ & $90(60.8)$ & $97(53.6)$ & $83(55.7)$ & $98(57.6)$ & $46(59.0)$ & $173(58.2)$ & $195(55.6)$ \\
\hline$\geq 21$ (Mild AD), $n(\%)$ & $93(32.5)$ & $134(35.4)$ & $58(39.2)$ & $84(46.4)$ & $66(44.3)$ & $72(42.4)$ & $32(41.0)$ & $124(41.8)$ & $156(44.4)$ \\
\hline $\begin{array}{l}\text { Years of exposure in parent } \\
\text { and extension studies }\end{array}$ & $1.41 \pm 0.828$ & $3.06 \pm 0.809$ & $1.33 \pm 0.756$ & $2.89 \pm 0.749$ & $1.30 \pm 0.741$ & $2.87 \pm 0.692$ & $3.16 \pm 0.781$ & $1.31 \pm 0.748$ & $2.88 \pm 0.721$ \\
\hline
\end{tabular}

$* \mathrm{~N}$ as of database cut-off date (represents a total of 1,390 subjects who received bapineuzumab in extension Study 351). Plus-minus values are means \pm SD. Years of exposure was calculated as the number of days from the day of the first infusion through either the day of the last infusion plus 137 days or the day of last study visit plus 1 day, whichever is shorter, divided by 365.25 . Subjects in the noncarrier study who were originally randomized to $2.0 \mathrm{mg} / \mathrm{kg}$ were reassigned to the $1.0 \mathrm{mg} / \mathrm{kg}$ dose in that study, and continued the $1.0 \mathrm{mg} / \mathrm{kg}$ dose in Study 351 are not included in the Bapi $1.0 \rightarrow 1.0 \mathrm{mg} / \mathrm{kg}$ group. APOE $\varepsilon 4$ denotes apolipoprotein $\mathrm{E}$, heterozygote, and homozygote denote 1 and 2 copy number of $\varepsilon 4$ allele. 
Table 2

Overview of adverse events for $A P O E \varepsilon 4$ carriers and noncarriers (safety analysis population)

\begin{tabular}{|c|c|c|c|c|}
\hline \multirow[t]{3}{*}{ Summary of Adverse Events } & \multirow{2}{*}{\multicolumn{2}{|c|}{$\begin{array}{c}A P O E \& 4 \text { Carriers } \\
\text { Study } 302 \rightarrow 351\end{array}$}} & \multirow{2}{*}{\multicolumn{2}{|c|}{$\begin{array}{c}\text { APOE } \varepsilon 4 \text { Noncarriers } \\
\text { Study } 301 \rightarrow 351 \\
\text { Pooled Doses }\end{array}$}} \\
\hline & & & & \\
\hline & $\begin{array}{c}\mathrm{pbo} \rightarrow \text { Bapi } \\
0.5 \mathrm{mg} / \mathrm{kg} \\
n(\%)\end{array}$ & $\begin{array}{l}\text { Bapi } 0.5 \rightarrow \\
0.5 \mathrm{mg} / \mathrm{kg} \\
n(\%)\end{array}$ & $\begin{array}{c}\mathrm{pbo} \rightarrow \text { Bapi } \\
n(\%)\end{array}$ & $\begin{array}{c}\text { Bapi } \rightarrow \text { Bapi } \\
n(\%)\end{array}$ \\
\hline Extension -Onset Adverse Events (through to final database lock) & $N=297$ & $N=391$ & $N=316$ & $\mathrm{~N}=380$ \\
\hline Participants who Died in the Study & $14(4.7)$ & $12(3.1)$ & $11(3.5)$ & $9(2.4)$ \\
\hline Any Treatment-Emergent AEs & $248(83.5)$ & $341(87.2)$ & $260(82.3)$ & $308(81.1)$ \\
\hline TEAEs Related to Study Drug & 79 (26.6) & $71(18.2)$ & $53(16.8)$ & $49(12.9)$ \\
\hline Any Treatment-Emergent SAEs & $89(30.0)$ & $92(23.5)$ & $80(25.3)$ & $73(19.2)$ \\
\hline TE SAEs Related to Drug & $13(4.4)$ & $11(2.8)$ & $7(2.2)$ & $12(3.2)$ \\
\hline \multicolumn{5}{|l|}{ Early Termination from Study due to } \\
\hline TEAEs & $40(13.5)$ & $45(11.5)$ & $31(9.8)$ & $31(8.2)$ \\
\hline TEAEs Related to Drug & $12(4.0)$ & $17(4.3)$ & $7(2.2)$ & $8(2.1)$ \\
\hline Parent and Extension Study Adverse Events (through to first database lock) ${ }^{\mathrm{a}}$ & $\mathrm{N}=286$ & $\mathrm{~N}=378$ & $\mathrm{~N}=297$ & $\mathrm{~N}=351$ \\
\hline Participants who Died in the Study & $12(4.2)$ & $11(2.9)$ & $7(2.4)$ & $6(1.7)$ \\
\hline Any Treatment-Emergent AEs & $275(96.2)$ & $373(98.7)$ & $288(97.0)$ & $337(96.0)$ \\
\hline TEAEs Related to Study Drug & $113(39.5)$ & $139(36.8)$ & $93(31.3)$ & $106(30.2)$ \\
\hline Any Treatment-Emergent SAEs & $95(33.2)$ & $135(35.7)$ & $88(29.6)$ & $103(29.3)$ \\
\hline TE SAEs Related to Drug & $10(3.5)$ & $13(3.4)$ & $9(3.0)$ & $15(4.3)$ \\
\hline \multicolumn{5}{|l|}{ Early Termination from Study due to } \\
\hline TEAEs & $34(11.9)$ & $39(10.3)$ & $31(10.4)$ & $27(7.7)$ \\
\hline TEAEs Related to Drug & $10(3.5)$ & $15(4.0)$ & $7(2.4)$ & $8(2.3)$ \\
\hline
\end{tabular}

${ }^{a}$ Overview of TEAEs that occurred in the parent and extension studies. Bapi, Bapineuzumab; pbo, Placebo; ARIA-E, amyloid-related imaging abnormalities that include intraparenchymal extravasation of fluid, sulcal effusions and gyral swelling that may or may not be present. Each AE was classified by the investigator as "Related" or "Not Related" AEs with missing relatedness are counted as "Related". Counts and percentages are based on the number of subjects with AEs (not events). Adverse events are coded using MedDRA version 14.1.

respectively) than in the bapi-bapi group (18.2\% and $23.5 \%$ ). No extension-onset AE occurred within 24 hours of an infusion. All AEs of ARIA-E were considered related to study drug. Overall, for both carriers and noncarriers in all treatment groups, the extension-onset AE profile and incidence proportions were similar to the AEs observed during the parent and extension studies.

Extension-onset TEAEs occurring in $\geq 5 \%$ of patients in any treatment group were nervous system and psychiatric disorders (Table 3). The most common AEs occurring in $\geq 10 \%$ of participants were fall, urinary tract infection, agitation, and ARIA-E. AEs with preferred terms categorized as ischemic stroke in the parent studies (e.g., cerebral infarction, transient ischemic attack, ischemic stroke) each occurred in $\leq 1.1 \%$ of participants in the pbo $\rightarrow$ bapi and bapi $\rightarrow$ bapi groups, in both carriers and noncarriers.

In both cohorts, a majority of TEAEs were rated mild or moderate. Among carriers, in newly exposed and previously exposed dose group, $15.8 \%$ and $14.6 \%$ experienced severe AEs, and 1.3 and $1.5 \%$ had life-threatening TEASs. Among noncarriers the corresponding percentages of those with severe AEs were $12.0 \%$ and $10.8 \%$ and those with lifethreatening AEs were $0.3 \%$ and $1.3 \%$ respectively. Within each cohort, the incidence of extensiononset SAEs was generally similar in the pbo $\rightarrow$ bapi and bapi $\rightarrow$ bapi groups, and most individual SAEs occurred in $<2 \%$ of patients in any treatment group. The incidence proportion of SAEs that occurred in $\geq 2 \%$ of carriers in either dose group were pneumonia (3.4\% and $1.0 \%$, respectively), convulsion ( $2.0 \%$ in each group) and syncope ( $2.0 \%$ and $1.8 \%$, respectively).

ARIA-E was reported as an SAE in 4 (1.3\%) carrier patients in the pbo $\rightarrow$ bapi group and none in the bapi $\rightarrow$ bapi group. The only SAE in $\geq 2 \%$ of non-carriers was pneumonia $(2.5 \%$ in pbo $\rightarrow$ bapi and $1.1 \%$ in bapi $\rightarrow$ bapi groups). ARIA-E as an SAE occurred in $2(0.6 \%)$ noncarrier patients in the pbo $\rightarrow$ bapi group and in $1(0.3 \%)$ in the bapi $\rightarrow$ bapi group. ARIA-E did not lead to study discontinuation in noncarriers, but in carriers the incidence proportion of ARIA-E leading to discontinuation was $2.1 \%$ in pbo $\rightarrow$ bapi and $1.3 \%$ in bapi $\rightarrow$ bapi dose groups. 
Table 3

Extension-onset treatment-emergent adverse events occurring in 5\% or more of patients in any treatment group for $A P O E \varepsilon 4$ carriers and noncarriers (safety analysis population)

\begin{tabular}{|c|c|c|c|c|}
\hline \multirow[t]{3}{*}{ Body System Preferred Term } & \multirow{2}{*}{\multicolumn{2}{|c|}{$\begin{array}{c}\text { APOE } \varepsilon 4 \text { Carriers } \\
\text { Study } 302 \rightarrow 351(\mathrm{~N}=688)\end{array}$}} & \multirow{2}{*}{\multicolumn{2}{|c|}{$\begin{array}{c}A P O E \varepsilon 4 \text { Noncarriers } \\
\text { Study } 301 \rightarrow 351 \\
\text { Pooled Doses }(\mathrm{N}=696) \\
\end{array}$}} \\
\hline & & & & \\
\hline & $\begin{array}{c}\text { pbo } \rightarrow \text { Bapi } \\
0.5 \mathrm{mg} / \mathrm{kg} \\
(\mathrm{N}=297) n(\%) \\
\end{array}$ & $\begin{aligned} & \text { Bapi } \\
& 0.5 \rightarrow 0.5 \mathrm{mg} / \mathrm{kg} \\
&(\mathrm{N}=391) n(\%) \\
&\end{aligned}$ & $\begin{array}{c}\mathrm{pbo} \rightarrow \text { Bapi } \\
(\mathrm{N}=316) \\
n(\%)\end{array}$ & $\begin{array}{c}\text { Bapi } \rightarrow \text { Bapi } \\
(\mathrm{N}=380) \\
n(\%) \\
\end{array}$ \\
\hline \multicolumn{5}{|l|}{ Patients with any TEAE } \\
\hline \multicolumn{5}{|l|}{ Gastrointestinal Disorders } \\
\hline Constipation & & & $9(2.8)$ & $21(5.5)$ \\
\hline Diarrhea & $24(8.1)$ & $26(6.6)$ & $20(6.3)$ & $19(5.0)$ \\
\hline Nausea & $18(6.1)$ & $19(4.9)$ & $16(5.0)$ & $15(3.9)$ \\
\hline Vomiting & $22(7.4)$ & $17(4.3)$ & $9(2.8)$ & $14(3.7)$ \\
\hline Gastroesophageal reflux dis. & & & $11(3.5)$ & $6(1.6)$ \\
\hline \multicolumn{5}{|c|}{ General Disorders and Administration Site Cond. } \\
\hline Asthenia & & & $7(2.2)$ & $4(1.0)$ \\
\hline Oedema peripheral & & & $9(2.8)$ & $15(3.9)$ \\
\hline \multicolumn{5}{|l|}{ Infections and Infestations } \\
\hline Cellulitis & & & $2(0.6)$ & $4(1.0)$ \\
\hline Nasopharyngitis & & & $17(5.4)$ & $17(4.5)$ \\
\hline Pneumonia & & & $12(3.8)$ & $10(2.6)$ \\
\hline Upper Respiratory Tract Infections & $12(4.0)$ & $20(5.1)$ & $10(3.2)$ & $22(5.8)$ \\
\hline Urinary tract infection & $37(12.5)$ & $49(12.5)$ & $34(10.8)$ & $39(10.3)$ \\
\hline \multicolumn{5}{|c|}{ Injury, Poisoning and Procedural Complications } \\
\hline Fall & $33(11.1)$ & $58(14.8)$ & $35(11.1)$ & $53(13.9)$ \\
\hline \multicolumn{5}{|l|}{ Metabolism and Nutrition Disorders } \\
\hline Dehydration & & & $9(2.8)$ & $15(3.9)$ \\
\hline Hypercholesterolemia & & & $1(0.3)$ & $5(1.3)$ \\
\hline Hypokalemia & & & $2(0.6)$ & $6(1.6)$ \\
\hline \multicolumn{5}{|c|}{ Musculoskeletal and Connective tissue Disorders } \\
\hline Arthralgia & $18(6.1)$ & $16(4.1)$ & $13(4.1)$ & $17(4.5)$ \\
\hline Back pain & $22(7.4)$ & $14(3.6)$ & $15(4.7)$ & $12(3.2)$ \\
\hline \multicolumn{5}{|l|}{ Neoplasm Benign or Malignant } \\
\hline Basal cell carcinoma & & & $10(3.2)$ & $3(0.8)$ \\
\hline \multicolumn{5}{|l|}{ Nervous System Disorders } \\
\hline Cerebral microhemorrhage & $22(7.4)$ & $9(2.3)$ & $12(3.8)$ & $7(1.8)$ \\
\hline Dementia Alzheimer's type & $16(5.4)$ & $17(4.3)$ & $10(3.2)$ & $11(2.9)$ \\
\hline Dizziness & $15(5.1)$ & $21(5.4)$ & $13(4.1)$ & $11(2.9)$ \\
\hline Headache & $29(9.8)$ & $29(7.4)$ & $18(5.7)$ & $21(5.5)$ \\
\hline Syncope & & & $10(3.2)$ & $12(3.2)$ \\
\hline ARIA-E & $35(11.8)$ & $20(5.1)$ & $17(5.4)$ & $5(1.3)$ \\
\hline \multicolumn{5}{|l|}{ Psychiatric Disorders } \\
\hline Agitation & $39(13.1)$ & $42(10.7)$ & $34(10.8)$ & $36(9.5)$ \\
\hline Anxiety & $21(7.1)$ & $35(9.0)$ & $22(7.0)$ & $15(3.9)$ \\
\hline Confusional state & $15(5.1)$ & $18(4.6)$ & & \\
\hline Depression & $25(8.4)$ & $24(6.1)$ & $29(9.2)$ & $19(5.0)$ \\
\hline Insomnia & $11(3.7)$ & $21(5.4)$ & & \\
\hline \multicolumn{5}{|l|}{ Renal and Urinary Disorders } \\
\hline Urinary incontinence & $15(5.1)$ & $18(4.6)$ & & \\
\hline \multicolumn{5}{|l|}{ Respiratory, Thoracic Disorder } \\
\hline Cough & & & $17(5.4)$ & $14(3.7)$ \\
\hline \multicolumn{5}{|l|}{ Vascular Disorders } \\
\hline Hypertension & & & $11(3.5)$ & $12(3.2)$ \\
\hline
\end{tabular}

Among carriers, there were 14 (4.7\%) deaths in the pbo $\rightarrow$ bapi and $12(3.1 \%)$ in the bapi $\rightarrow$ bapi group, and among noncarriers death occurred in $11(3.5 \%)$ and $9(2.4 \%)$ of patients in the two dose groups respectively. This represented an increase in the total number of deaths ( 3 among carriers and 9 among noncarriers) compared with the parent studies. Two additional deaths occurred after the last dose of study drug. Both were noncarriers: a 61-year-old male who received bapineuzumab $1.0 \mathrm{mg} / \mathrm{kg}$ in parent Study 
Table 4

Incidence proportion of extension-onset treatment-emergent adverse events of special circumstance for $A P O E \varepsilon 4$ carriers and noncarriers (safety analysis population)

\begin{tabular}{|c|c|c|c|c|}
\hline \multirow[t]{3}{*}{$\begin{array}{l}\text { Treatment-Emergent Adverse } \\
\text { Events of Special Circumstance }\end{array}$} & \multirow{2}{*}{\multicolumn{2}{|c|}{$\begin{array}{c}\text { APOE } \varepsilon 4 \text { Carriers } \\
\text { Study } 302 \rightarrow 351(N=688)\end{array}$}} & \multicolumn{2}{|c|}{$\begin{array}{c}A P O E \varepsilon 4 \text { Noncarriers } \\
\text { Study } 301 \rightarrow 351\end{array}$} \\
\hline & & & \multicolumn{2}{|c|}{ Pooled Doses $(\mathrm{N}=696)$} \\
\hline & $\begin{array}{c}\mathrm{pbo} \rightarrow \text { Bapi } \\
0.5 \mathrm{mg} / \mathrm{kg} \\
(\mathrm{N}=297) \\
\end{array}$ & $\begin{array}{c}\text { Bapi } 0.5 \rightarrow \\
0.5 \mathrm{mg} / \mathrm{kg} \\
(\mathrm{N}=391)\end{array}$ & $\begin{array}{c}\text { pbo } \rightarrow \text { Bapi } \\
(\mathrm{N}=316)\end{array}$ & $\begin{array}{l}\text { Bapi } \rightarrow \text { Bapi } \\
\quad(\mathrm{N}=380)\end{array}$ \\
\hline ARIA-E, $n$ & 35 & 20 & 17 & 5 \\
\hline$\%(95 \% \mathrm{CI})$ & $11.8(8.3,16.0)$ & $5.1(3.2,7.8)$ & $5.4(3.2,8.5)$ & $1.3(0.4,3.0)$ \\
\hline Difference $^{\mathrm{a}} \%(95 \% \mathrm{CI})$ & & $-6.7(-11.2,-2.1)$ & & $-4.1(-7.1,-1.0)$ \\
\hline Intracranial Hemorrhage, $n$ & 4 & 5 & 6 & 4 \\
\hline$\%(95 \% \mathrm{CI})$ & $1.3(0.4,3.4)$ & $1.3(0.4,3.0)$ & $1.9(0.7,4.1)$ & $1.1(0.3,2.7)$ \\
\hline Difference $^{\mathrm{a}} \%(95 \% \mathrm{CI})$ & & $-0.1(-2.1,1.9)$ & & $-0.8(-3.0,1.3)$ \\
\hline Seizures/Convulsions, $n$ & 6 & 10 & 4 & 7 \\
\hline$\%(95 \% \mathrm{CI})$ & $2.0(0.7,4.3)$ & $2.6(1.2,4.7)$ & $1.3(0.3,3.2)$ & $1.8(0.7,3.8)$ \\
\hline Difference $^{\mathrm{a}} \%(95 \% \mathrm{CI})$ & & $0.5(-2.0,3.1)$ & & $0.6(-1.5,2.7)$ \\
\hline Deep Vein Thrombosis/Pulmonary Embolism, $n$ & 3 & 3 & 2 & 7 \\
\hline$\%(95 \% \mathrm{CI})$ & $1.0(0.2,2.9)$ & $0.8(0.2,2.2)$ & $0.6(0.1,2.3)$ & $1.8(0.7,3.8)$ \\
\hline Difference $^{\mathrm{a}} \%(95 \% \mathrm{CI})$ & & $-0.2(-2.0,1.5)$ & & $1.2(-0.7,3.1)$ \\
\hline
\end{tabular}

${ }^{a}$ Incidence proportion is the ratio of the number of subjects with the event to the total number of subjects $* 100$. The exact binomial $95 \% \mathrm{CI}$ is calculated for each treatment group proportion and for the difference in treatment proportions. Extension-onset AEs are treatment-emergent AEs which started during or after the first bapineuzumab infusion in the extension study and prior to or on the date of last dose +137 days.

301 , and 5 doses in the extension study died due to progressive dementia and a 76-year-old male who had received bapineuzumab $0.5 \mathrm{mg} / \mathrm{kg}$ in Study 301 and 9 doses in the extension study died due to prostate cancer ( $>137$ days after the last dose).

Events of special circumstance (ESCs) were AEs that were prespecified to be reported to the sponsor regardless of the investigators' assessment of seriousness or causality. For bapineuzumab IV ARIA-E, cerebral hemorrhage, seizures or convulsions, and pulmonary embolism were identified as ESC per protocol. Their incidence proportion and the difference between the pbo $\rightarrow$ bapi and bapi $\rightarrow$ bapi dose groups are shown in Table 4. The incidence of ARIA-E was higher among carriers than noncarriers over the entire parent plus extension studies. ARIA-E occurred in $12.2 \%$ of carriers versus $5.4 \%$ of noncarriers for the pbo $\rightarrow$ bapi group and for the bapi $\rightarrow$ bapi group $11.9 \%$ versus $6.0 \%$ developed ARIA-E respectively. Extension-onset ARIA-E occurred in $11.8 \%$ carriers and $5.4 \%$ noncarriers in pbo $\rightarrow$ bapi group, versus $5.1 \%$ and $1.3 \%$ in bapi $\rightarrow$ bapi group, respectively.

In noncarriers, there was a dose dependent increase in the incidence proportion of ARIA-E among patients who were newly exposed to bapineuzumab $(3.2 \%$ in $\mathrm{pbo} \rightarrow$ bapi $0.5 \mathrm{mg} / \mathrm{kg}$, and $7.6 \%$ in pbo $\rightarrow$ bapi $1.0 \mathrm{mg} / \mathrm{kg}$ ) but not among those previously exposed group. There were no noteworthy differences in the incidence proportions of intracranial hemorrhage, or DVT/PE between carriers and noncarriers, or between the pbo $\rightarrow$ bapi and bapi $\rightarrow$ bapi groups within each cohort. The small difference in incidence of seizures/convulsion in carriers versus noncarriers who received bapineuzumab in both the parent and extension studies $(2.6 \%$ versus $1.8 \%)$ was not clinically relevant due to a small number of patients who experienced seizure/convulsions.

In both carriers and noncarriers who first received bapineuzumab in the extension study (i.e., pbo $\rightarrow$ bapi group), all cases of ARIA-E began after the 1st, 2nd, or 3rd bapineuzumab infusion.

ARIA-E was asymptomatic in most cases. Clinical observations solicited from investigators associated with cases of ARIA-E included altered mental status (e.g., increased confusion, lethargy, disorientation or hallucinations), seizures, gait difficulties, vomiting, headache fatigue, and dizziness. In carriers, the cases of symptomatic extension-onset ARIA-E in the pbo $\rightarrow$ bapi group all occurred after first dose $(n=1)$, dose $2(n=5)$ or dose $3(n=1)$, which was similar to the pattern in parent studies. The single case of symptomatic ARIA-E in the bapi $\rightarrow$ bapi group occurred after dose 8 . In noncarriers, all cases of symptomatic ARIA-E occurred after the first dose of bapineuzumab. Asymptomatic ARIA-E occurred after the first three doses of bapineuzumab in the pbo $\rightarrow$ bapi group $(n=12)$ and after doses 5 and 9 in the bapi $\rightarrow$ bapi group $(n=3)$. 
Clinical severity of ARIA-E was rated by the investigator as mild or moderate for all cases in carriers (except in one) and noncarriers. Clinical severity in one carrier, in the pbo $\rightarrow$ bapi group, was rated severe (dizziness). Extension-onset ARIA$E$ resolved without sequelae in most patients. In carriers, extension-onset ARIA-E resolved in 32 of 34 in the pbo $\rightarrow$ bapi group and in 17 of 19 in the bapi $\rightarrow$ bapi group. ARIA-E in 1 carrier in the bapi $\rightarrow$ bapi group resolved with sequelae (two ongoing events of cerebral microhemorrhage and hemosiderin deposits $\geq 10 \mathrm{~mm}$ in size) and residual cerebral edema did not recede in 1 carrier (in pbo $\rightarrow$ bapi group) at the last follow-up MRI visit. In noncarriers, all cases of extension-onset ARIA-E resolved. The median duration of cases that resolved was 79 days and 77 days for carriers in the pbo $\rightarrow$ bapi and bapi $\rightarrow$ bapi early groups respectively and for noncarriers it was 93 and 107 days, respectively.

At extension baseline (i.e., the last MRI Central Read prior to the first dose of bapineuzumab in Study 351), a numerically higher percentage of patients in the previously exposed bapi $\rightarrow$ bapi group were identified with small hemosiderin deposits (HDs, $<10 \mathrm{~mm}$; synonymous with microhemorrhages) (carriers: $15.9 \%$, noncarriers $14.5 \%$ ) versus those in the previously unexposed pbo $\rightarrow$ bapi group (carriers: $10.5 \%$; noncarriers: $10.1 \%$ ). This was expected given the screening procedures for Study 351. If a participant who wanted to enter the extension study had a significant number of HDs $<10 \mathrm{~mm}$ on the last MRI, the brain MRI worksheet was sent to an unblinded monitor. If the participant had received bapineuzumab during the parent study, he or she was permitted to enroll in Study 351, but if received placebo during the parent study, he or she was not permitted to enroll. Approximately $1 / 3$ of the carriers had HDs prior to the onset of the first ARIA-E, approximately half of them had HDs at onset and at the end of an episode of ARIA-E, and approximately $40 \%$ of them had HDs after ARIA-E resolved. The results were similar in the pbo $\rightarrow$ bapi and bapi $\rightarrow$ bapi groups.

Patients who experienced intracranial hemorrhage in either parent study or the extension study discontinued treatment as per sponsor's recommendation. Nonetheless, a few patients who were identified with intracranial hemorrhage in the parent study participated in the extension study. Intracranial hemorrhage had occurred in 3 carriers and 3 noncarriers during the parent study (in both, 1 in pbo $\rightarrow$ bapi and 2 in bapi $\rightarrow$ bapi group). Among carriers, extension-onset intracranial hemorrhage occurred in 4 patients in the newly exposed group (2 with subdural hematoma, 1 with cerebellar hemorrhage, and 1 with subarachnoid hemorrhage) and in 5 patients in the previously exposed group (4 with subdural hematoma, and 1 with subarachnoid hemorrhage). Subdural hematoma led to death in 1 patient in the pbo $\rightarrow$ bapi group approximately 6 months after onset. Among noncarriers, intracranial hemorrhage occurred in 6 patients in the pbo $\rightarrow$ bapi group ( 3 with subdural hematoma and 3 with cerebral hemorrhage) and in 4 in the bapi $\rightarrow$ bapi group (all with subdural hematoma). Subdural hematoma led to death in one patient diagnosed in each dose group.

\section{Clinical efficacy}

Table 5 shows the MMRM analysis of leastsquares (LS) means (SE), and the LS mean difference between pbo $\rightarrow$ bapi and bapi $\rightarrow$ bapi dose groups for change in ADAS-Cog/11 and DAD total scores from parent baseline to extension week 65 in the parent mITT population. There was no statistically significant difference in ADAS-Cog/11 and DAD total scores between participants who had received bapineuzumab since the beginning of the parent study (early start dose group) and those who first received bapineuzumab during the extension studies (delayed start dose group) in either carriers or noncarriers.

Efficacy results from the extension study indicated that lack of effect on clinical cognitive and functional efficacy endpoints persisted after long-term treatment with bapineuzumab IV therapy. A subgroup analysis by disease severity showed similar results for participants with either mild or moderate AD. No changes in ADAS-Cog/11 and DAD total scores were observed in any bapineuzumab treatment cohort during the extension study (from extension baseline to extension week 65). The trajectories of observed values for ADAS-Cog/11 and DAD over the entire course of the parent and extension studies (to week 65) are presented in Figs. 2 and 3, respectively, and show that both carries and noncarriers continued to decline in cognition and function.

\section{Exploratory efficacy, health outcome, and biomarker assessments}

The results of MMRM analysis of change from parent baseline to extension week 65 for MMSE total score and the following health outcomes of Dependence Scale total score, NPI total score, Abbr. 
Table 5

ADAS-Cog/11, DAD total scores: Change from parent baseline to extension Week 65 for APOE $\varepsilon 4$ carriers and noncarriers (parent study mITT population)

\begin{tabular}{|c|c|c|c|c|}
\hline \multirow[t]{2}{*}{ Secondary efficacy objective time point } & \multicolumn{2}{|c|}{ Study $302 A P O E \varepsilon 4$ Carriers } & \multicolumn{2}{|c|}{ Study 301 Noncarriers (Pooled doses) } \\
\hline & $\begin{array}{c}\mathrm{pbo} \rightarrow \text { Bapi } \\
0.5 \mathrm{mg} / \mathrm{kg} \\
(\mathrm{N}=432)\end{array}$ & $\begin{array}{c}\text { Bapi } 0.5 \rightarrow \\
0.5 \mathrm{mg} / \mathrm{kg} \\
(\mathrm{N}=658)\end{array}$ & $\begin{array}{c}\text { pbo } \rightarrow \text { Bapi } \\
(\mathrm{N}=493)\end{array}$ & $\begin{array}{c}\text { Bapi } \rightarrow \text { Bapi } \\
(N=621)\end{array}$ \\
\hline \multicolumn{5}{|l|}{ ADAS-Cog/11 Total Scores } \\
\hline \multicolumn{5}{|c|}{ Change from Parent Baseline at Extension Week 65} \\
\hline$n$ & 148 & 209 & 145 & 187 \\
\hline Mean (SD) & $13.8(13.00)$ & $12.7(12.50)$ & $11.8(13.39)$ & $8.2(11.90)$ \\
\hline \multicolumn{5}{|l|}{ MMRM Analysis at Extension Week $65^{\mathrm{a}}$} \\
\hline LS Mean (SE) & $17.8(0.82)$ & $17.2(0.68)$ & $15.5(0.81)$ & $15.0(0.72)$ \\
\hline Difference of LS Means $(95 \% \mathrm{CI})^{\mathrm{b}}$ & & $-0.6(-2.7,1.5)$ & & $-0.5(-2.6,1.6)$ \\
\hline$p$-value & & 0.568 & & 0.667 \\
\hline \multicolumn{5}{|l|}{ DAD Total Scores } \\
\hline \multicolumn{5}{|c|}{ Change from Parent Baseline at Extension Week 65} \\
\hline$n$ & 153 & 207 & 147 & 189 \\
\hline Mean (SD) & $-24.5(24.28)$ & $-24.6(24.26)$ & $-23.4(23.45)$ & $-18.2(22.09)$ \\
\hline \multicolumn{5}{|l|}{ MMRM Analysis at Extension Week $65^{\mathrm{a}}$} \\
\hline LS Mean (SE) & $-31.5(1.67)$ & $-33.2(1.43)$ & $-28.3(1.57)$ & $-28.2(1.40)$ \\
\hline Difference of LS Means $(95 \% \mathrm{CI})^{\mathrm{b}}$ & & $-1.7(-6.0,2.6)$ & & $0.1(-4.0,4.2)$ \\
\hline$p$-value & & 0.430 & & 0.964 \\
\hline
\end{tabular}

${ }^{\text {a }}$ Results were from a restricted maximum likelihood (REML)-based mixed model for repeated measures (MMRM) with change from parent baseline as the response variable and the fixed effect model terms for treatment, visit (scheduled week), treatment-by-visit interaction, baseline value, baseline value-by-visit interaction, MMSE total score stratum, cholinesterase inhibitor or memantine use stratum, apolipoprotein $(A P O E) \varepsilon 4$ allele copy number stratum, and baseline age. ${ }^{\mathrm{b}}$ Treatment differences (Study 302: Bapi $0.5 \rightarrow 0.5 \mathrm{mg} / \mathrm{kg} \mathrm{minus} \mathrm{Pbo} \rightarrow \mathrm{Bapi}$ $0.5 \mathrm{mg} / \mathrm{kg}$; Study 301:Bapi $\rightarrow$ Bapi minus Pbo $\rightarrow$ Bapi) were estimated using least-squares (LS) means with factor levels weighted according to overall baseline sample proportions. Bapi = Bapineuzumab; pbo = Placebo. The ADAS-Cog/11 total score ranges from 0 (less impairment) to 70 (greater impairment); a positive change from baseline indicates worsening. DAD total score ranges from 0 (greater impairment) to 100 (less impairment); a negative change from baseline indicates worsening.

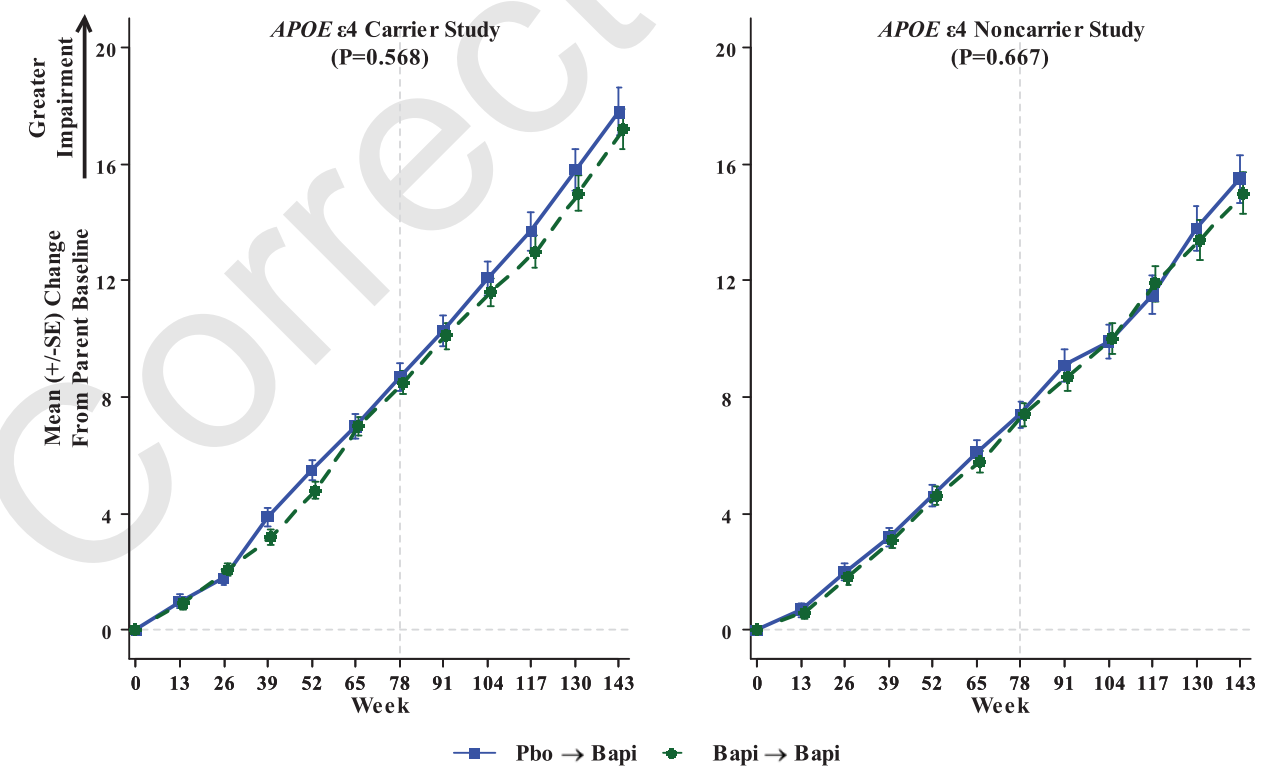

Fig. 2. ADAS-Cog/11 total score change over time from parent baseline to extension Week 65 for APOE $\varepsilon 4$ carriers and noncarriers (parent study mITT population).

RUD-Lite Primary Caregiver total time per month, and HUI total score are shown in Supplementary Table 1. For all efficacy and health outcome measures, the LS mean difference between the pbo $\rightarrow$ bapi and the bapi $\rightarrow$ bapi pooled dose groups was not statistically significant at any point over the duration of 

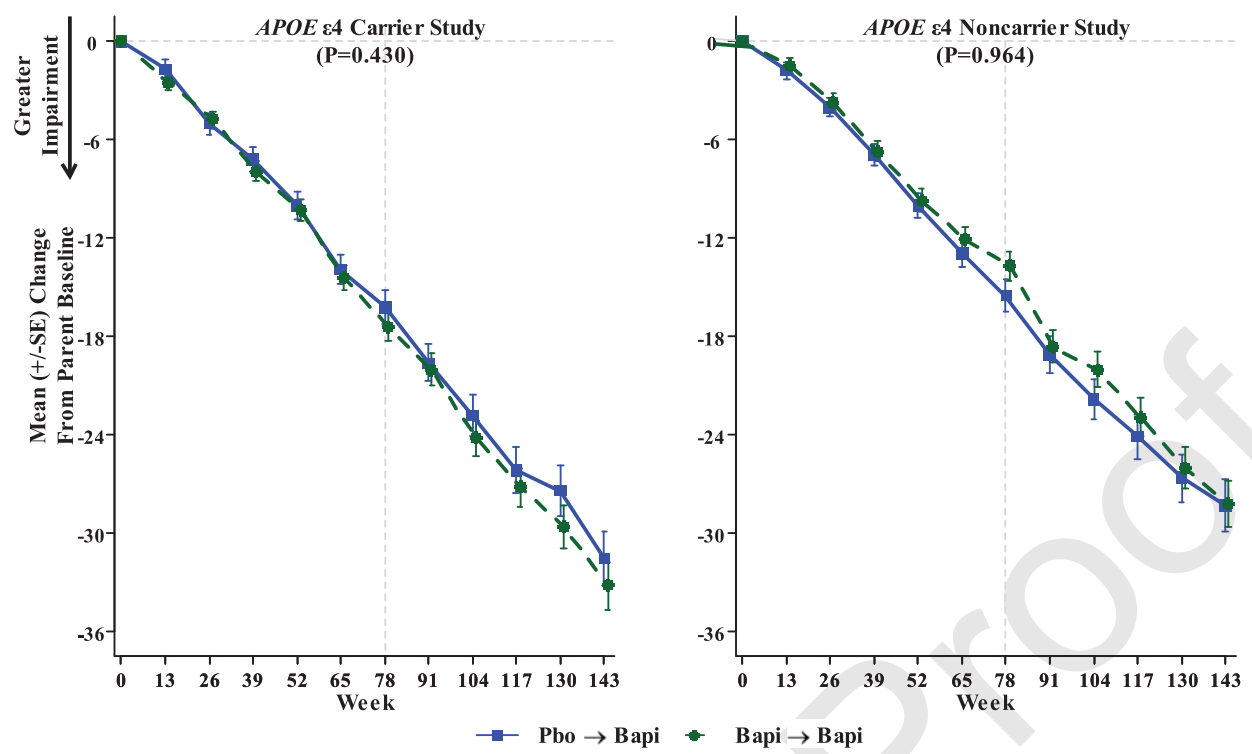

Fig. 3. DAD total score change over time from parent baseline to extension Week 65 for APOE $\varepsilon 4$ carriers and noncarriers (parent study mITT population).

parent and extension studies in either carriers or noncarriers. Time course of mean change in total scores for NPI and Abbr. RUD-Lite Primary Caregiver total time per month are shown in Supplementary Figures 1 and 2. Bapineuzumab therapy did not result in any improvement in the key health outcome endpoints

Only a small number of carriers $(n=39)$ and noncarriers $(n=5)$ participated in the PiB PET imaging substudy and were followed for evaluation of change in brain amyloid burden from parent baseline to extension study Week 52, thus no MMRM analyses were performed. There was no meaningful change from parent study baseline to Week 52 of extension study in the SUVr for the global cortical average for pbo $\rightarrow$ bapi and bapi $\rightarrow$ bapi dose groups in either carriers or noncarriers (Supplementary Table 2).

Brain volume changes overtime from parent study baseline to extension study Week 45 and 65 for BBSI, VBSI, WBV, VV, and HBSI measured by vMRI with the MMRM analyses are shown in Supplementary Table 3. The least square mean difference in brain volume change between the pbo $\rightarrow$ bapi group and bapi $\rightarrow$ bapi group was not statistically significant at either time point for either carriers or noncarriers except for right HBSI. The latter showed a statistically significant shift from parent baseline to extension week 45 between the delayed start and early start dose groups for carriers $(p=0.014)$, but not for noncarriers (0.863). Change from baseline in BBSI
vMRI at several time points over the entire duration of parent and extension study is illustrated in Fig. 4.

The relationships between change from parent baseline to extension Week 65 in vMRI BBSI (mL/year) and changes in cognitive and functional measures (ADAS-Cog/11 and DAD total scores) were determined in the vMRI analysis population. In carriers and noncarriers, vMRI BBSI at extension week 65 was significantly positively correlated with change in ADAS-Cog scores for the pbo $\rightarrow$ bapi and bapi $\rightarrow$ bapi dose groups, indicating cognitive decline was associated with greater brain volume loss. In carriers, the Pearson correlation coefficients for BBSI (mL/year) at extension Week 65 versus change in ADAS-Cog score was 0.505 for the pbo $\rightarrow$ bapi group and 0.670 for the bapi $\rightarrow$ bapi group ( $p$-values $<0.001$ ). Similarly, in noncarriers, the Pearson correlation coefficients were 0.683 for the pbo $\rightarrow$ bapi group and 0.642 for the bapi $\rightarrow$ bapi group ( $p$-values $<0.001$ ).

Change in vMRI BBSI (mL/year) was weakly negatively correlated with change in DAD total score for the pbo $\rightarrow$ bapi and bapi $\rightarrow$ bapi dose groups in both carriers and noncarriers, indicating functional decline was not correlated with greater volume reduction. In carriers, the Pearson correlation coefficients for BBSI at extension Week 65 versus change in DAD total score was -0.364 ( $p$-value $=0.014$ ) for the pbo $\rightarrow$ bapi group and -0.530 for the bapi $\rightarrow$ bapi group ( $p$-values $<0.001$ ). 

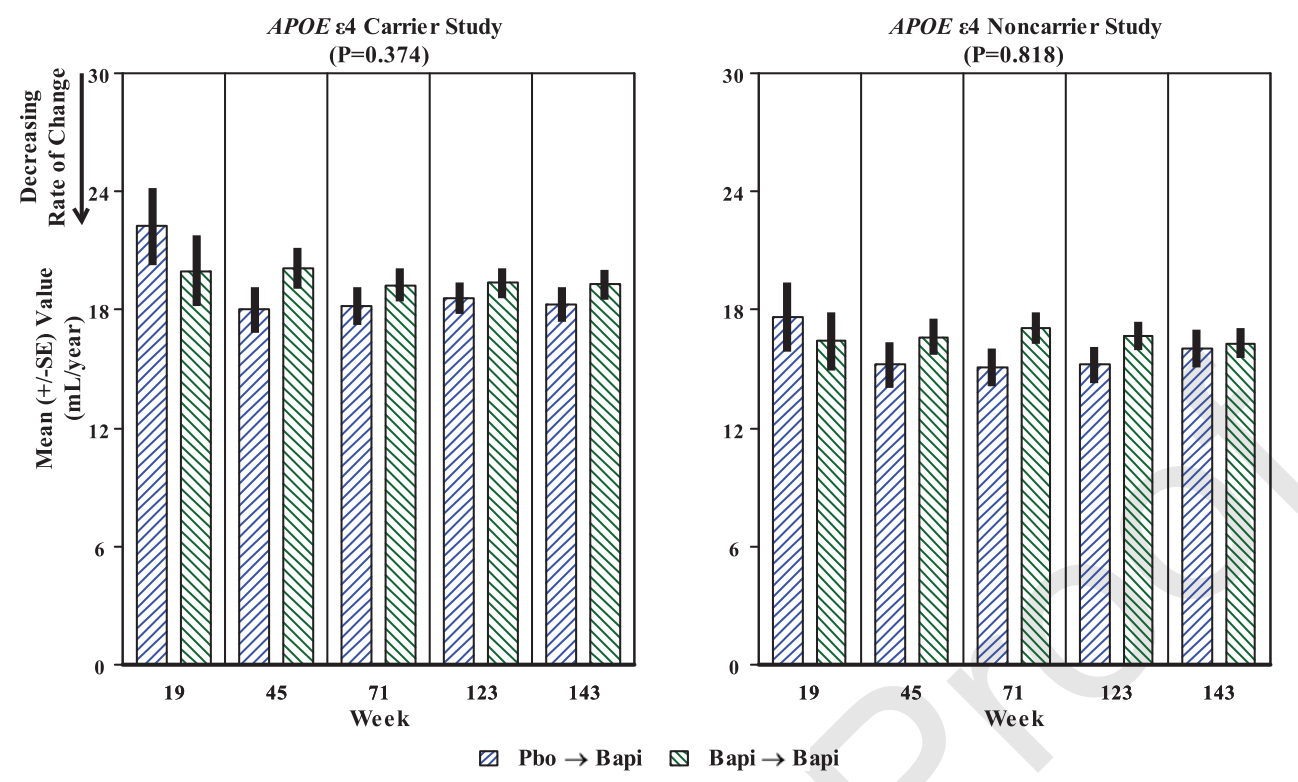

Fig. 4. vMRI brain boundary shift integral observed values over time (mL/year) (mixed model for repeated measures analysis, vMRI population).

In noncarriers, corresponding correlation coefficients were $-0.291 \quad(p$-value $=0.158)$ and -0.584 $(p$-value $<0.001)$, respectively.

Change over time from parent study baseline in CSF level of p-tau to extension Week 65 is shown in Fig. 5, and there was no significant difference between the newly and previously exposed groups for either carriers or noncarriers. Similar findings were observed for changes in the CSF levels of Ttau, and $A \beta\left(A \beta_{x-40}\right)$, except for change in CSF level of $A \beta_{x-42}$ between the two dose groups which was statistically significant in carriers only $(p=0.005)$ (Supplementary Table 2).

\section{DISCUSSION}

We reported on long-term safety of bapineuzumab IV administered every 13 weeks over approximately 3 years in patients with mild-to-moderate AD who had completed their participation in the original double-blind parent studies (Study 301 and Study 302) [3] and were enrolled into the phase III extension study.

Infusion of bapineuzumab 0.5 or $1.0 \mathrm{mg} / \mathrm{kg}$ every 13 weeks was generally well tolerated, with a safety profile that was similar to the parent studies [3]. A noteworthy difference was that incidence proportion of extension-onset ARIA-E in both noncarriers and carriers who first received bapineuzumab in the extension study was higher than that in those who received bapineuzumab in the preceding double-blind study. This finding is consistent with a reduction in risk for ARIA-E with prior exposure to bapineuzumab. The frequency of extension-onset ARIA-E in the pbo $\rightarrow$ bapi group (pbo $\rightarrow$ bapi: carriers; $11.8 \%$, noncarriers, $5.4 \%$ ) was comparable to the incidence of ARIA-E detected during the doubleblind parent studies in the MRI Safety Read (carriers, $15.3 \%$; noncarriers pooled 0.5 and $1.0 \mathrm{mg} / \mathrm{kg}, 6.8 \%$ ) [12].

This higher incidence proportion of ARIA-E in patients who first received bapineuzumab in the extension study compared with those who had previously received bapineuzumab in the parent studies, confirms the decline in the incidence of ARIA-E over time with continued bapineuzumab exposure, and reduced risk of ARIA-E after longer exposure. In both carriers and noncarriers, all cases of extensiononset ARIA-E and most cases of HD began after the first, second or third bapineuzumab infusion. This is consistent with the finding in parent studies that detected a higher frequency of first episodes of ARIA-E during the intervals between first and third bapineuzumab infusions followed by decline after subsequent infusions [12]. ARIA-E was generally mild, asymptomatic, and resolved without sequelae. ARIA-E in 1 carrier in the bapi $\rightarrow$ bapi group resolved with sequelae and did not resolve in 4 carriers. Changes in MMSE and ADAS-Cog/11 scores from the visit preceding ARIA-E onset to 

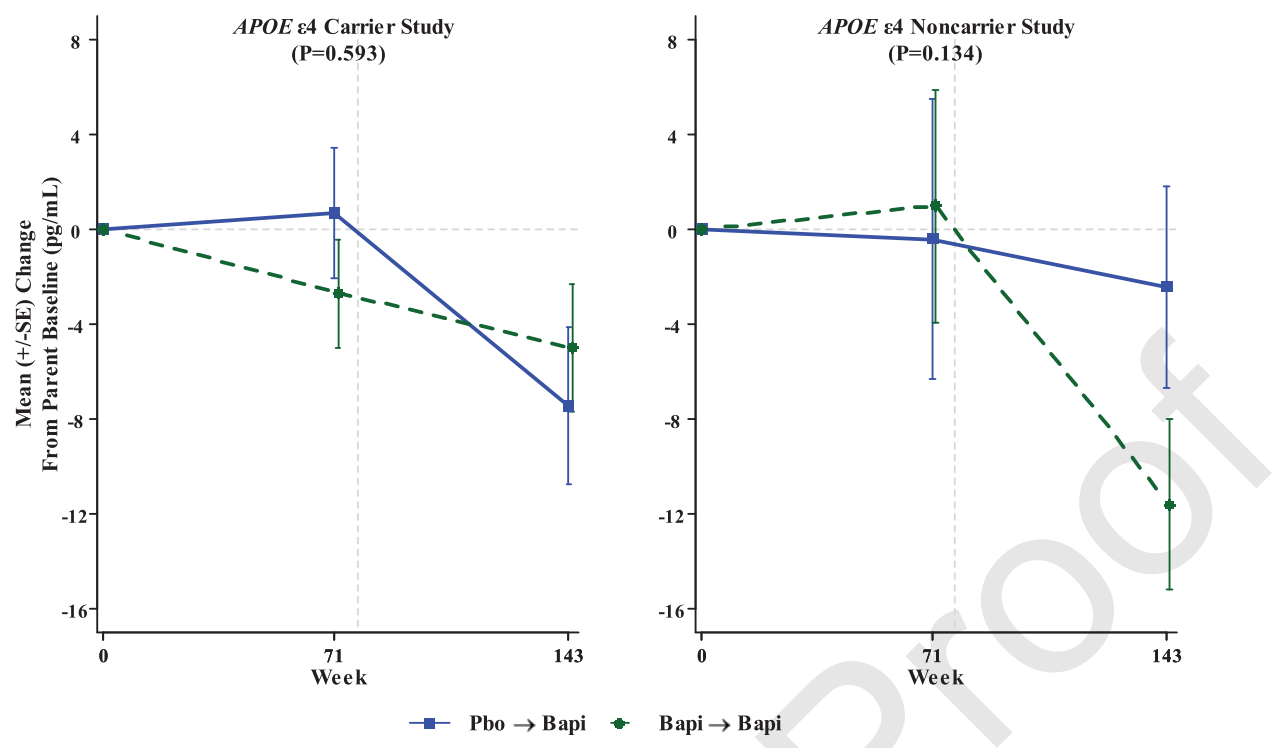

Fig. 5. Cerebrospinal fluid level of phosphorylate tau over time ( $\mathrm{pg} / \mathrm{mL})$ (mixed model for repeated measures analysis, CSF population). Number of observed values at baseline, Weeks 71 and 143: for $A P O E \varepsilon 4$ carriers: pbo $\rightarrow$ bapi $n=22$, 21, and 19 respectively; for bapi $\rightarrow$ bapi $n=31,29$ and 28 respectively; for noncarriers pbo $\rightarrow$ bapi $n=15,15$, and 15 respectively; for bapi $\rightarrow$ bapi $n=21,21$ and 20 , respectively.

the following visit were small, similar in noncarriers and carriers, and similar within each cohort in the placebo $\rightarrow$ bapi and bapi $\rightarrow$ bapi groups. It is difficult to draw conclusions about lack of ARIA-E effect on cognition in the extension study due to the decreased MRI frequency and relatively small proportion of subjects with extension-onset of ARIA-E compared with the parent studies, and the overall cognitive decline across all groups. However, clinical correlates of ARIA-E in the blinded phase III parent studies indicated no difference in the rate of decline on ADAS-Cog, MMSE or DAD assessments in bapineuzumab-treated groups with ARIA-E as compared with non-ARIA-E participants [14].

Based on extension-onset AEs, the long-term safety and tolerability profile of bapineuzumab IV was generally similar in $A P O E \varepsilon 4$ carriers and noncarriers except for a higher incidence proportion of ARIA-E in carriers versus noncarriers within the respective pbo $\rightarrow$ bapi and bapi $\rightarrow$ bapi dose groups. In the PiB PET substudies of parent phase III trials, $36.1 \%(21 / 61)$ of noncarriers and only $6.5 \%$ (8/123) of carriers tested negative for amyloid at baseline (i.e., had SUVR <1.35) and were excluded from the analyses of change from baseline in brain amyloid signal [3, 9]. Hence, differences in incidence of ARIA-E among carriers and noncarriers may in part be attributed to higher prevalence of significant amyloid burden at baseline in the carrier group as compared to noncarriers and to differences in mechanism of amyloid-clearance related to $A P O E \varepsilon 4$ in carriers. The frequency of TEAEs in both pbo $\rightarrow$ bapi and bapi $\rightarrow$ bapi dose groups is consistent with the long duration of this study and an elderly AD population and were comparable to frequency of $\mathrm{AE}$ events reported in the parent studies [3]. The percentages of patients with SAEs were higher for carriers than noncarriers. In both $A P O E \varepsilon 4$ carriers and noncarriers, no individual treatment-emergent SAE occurred with a frequency of $>3.5 \%$ in either treatment group and most occurred in $<2 \%$ of patients. The SAE profiles were generally similar between carriers and noncarriers and within each cohort percentages of patients with individual SAEs were similar in the two treatment groups. There were no noteworthy differences between $A P O E \varepsilon 4$ noncarriers and carriers, or between the dose groups within each cohort, in the incidence proportions of other ESCs, i.e., intracranial hemorrhage or DVT/PE.

The double-blind, placebo-controlled parent studies that preceded this long-term extension study did not show statistically significant differences between bapineuzumab IV and placebo in either cognitive or functional clinical endpoints [3]. After 6 to 12 months of bapineuzumab treatment in the extension study, there was a lack of treatment difference in the efficacy endpoints between patients who previously received bapineuzumab in parent studies prior to extension 
study and those who had received placebo prior to the extension study. This indicated there was no evidence of delayed benefit from previous exposure to bapineuzumab.

Relative to the baseline in parent Study 301 or 302 , change in ADAS-Cog/11 total score at extension Week 65, was numerically in favor of less cognitive impairment for the bapi $\rightarrow$ bapi group as compared to $\mathrm{pbo} \rightarrow$ bapi group, but was not statistically significant (in carriers, $p=0.568$; in noncarriers $p=0.667$ ). Bapineuzumab treatment over time did not result in any significant improvement in key health outcome endpoints and progressive decline continued despite treatment, indicating that long-term treatment with bapineuzumab at these doses did not slow AD progression. The lack of clinical benefit may in part be explained by insufficient amyloid clearing at given doses which were limited by treatment-emergent ARIA-E, inadequately altering accumulation of the most pathologic $A \beta$ species, and by initiating bapineuzumab treatment too late in the disease process. The long preclinical phase to AD involves not only amyloid deposition but also progressive and likely irreversible neurodegeneration with brain volume loss $[15,16]$; this process gradually accelerates and may therefore be more difficult to slow after emergence of clinical signs of AD dementia [17].

Longitudinal studies of other anti-A $\beta$ monoclonal antibodies targeting amyloid pathophysiologic processes in patients with mild-to-moderate $\mathrm{AD}$ attempted to reduce brain amyloid burden by triggering its clearance and diminishing its neurotoxicity, but there was no slowing of progression of AD. These other monoclonal antibodies include solanezumab which binds with soluble $A \beta$ [18], gantenerumab which binds with monomeric and fibrillar $A \beta$ [19], and aducanumab which selectively targets aggregated $\mathrm{A} \beta$. However, treatment with aducanumab in patients with prodromal or mild AD slowed decline of clinical measures (CDR-SB and MMSE scores) [20] and is being evaluated in early AD (NCT02477800). Intravenous immunoglobulin antibodies purported to increase efflux of aggregated $A \beta$ species from the brain did not improve cognition or function at 18 months in patients with mild-to-moderate AD [21]. Two ongoing global studies, ENGAGE (NCT02477800) and EMERGE (NCT02484547), are evaluating the safety and effectiveness of aducanumab in delaying cognitive impairment and disability in people with early AD.

Other reasons for lack of clinical efficacy of antiamyloid therapies may be due to treating patients who already have substantial neurodegeneration and cognitive impairment, or including patients without significant amyloid deposition $[18,19]$. Hence, proposed treatment strategies to modify AD symptoms and course of the disease have included targeting elevated levels of brain amyloid in presymptomatic stages of $\mathrm{AD}$, combined therapy for amyloid plaques and neurofibrillary tangles, and better management of related risk factors such as vascular disease in asymptomatic persons [22-25]. The A4 Study (NCT02008357) was designed with the aim of providing further insight into research questions of whether effectiveness of anti-amyloid therapy is limited by insufficient dose and not being administered early enough [26]. That study is investigating whether solanezumab treatment can slow progression of clinical symptoms of $\mathrm{AD}$ in older individuals who have evidence of cerebral amyloid accumulation. The study is currently ongoing, and findings are expected in 2022.

The results of the PiB PET, vMRI, and CSF substudies were similar for noncarriers and carriers. Within each cohort, statistically significant differences between the pbo $\rightarrow$ bapi group and bapi $\rightarrow$ bapi group were infrequent and not consistent. Overall, these findings indicate that a longer duration of bapineuzumab therapy at the delivered doses had no effect on biomarkers of AD pathology. However, the change in CSF level of $A \beta_{42}$ between the two dose groups was statistically significant in carriers only $(p=0.005)$. In the parent studies, the reduction from baseline in CSF p-tau was significantly greater for bapineuzumab $0.5 \mathrm{mg} / \mathrm{kg}$ versus placebo in carriers and for the $1.0 \mathrm{mg} / \mathrm{kg}$ dose in noncarriers. In this analysis, the fact that CSF p-tau levels in the pbo $\rightarrow$ bapi group were not different from the bapi $\rightarrow$ bapi group is noteworthy and suggests that dosing can be delayed and CSF p-tau reduction can "catch-up" to the previously exposed group.

In the PET substudies of parent studies, $36 \%$ of noncarriers and $5 \%$ of carriers had SUVrs below the specified threshold for cerebral amyloid signal [3]. Although challenges remain with relating AD disease biomarkers to different aspects of underlying $\mathrm{AD}$ pathology, enrolling patients with early stage AD disease confirmed by biomarker verification may be more effective in future trials.

A limitation of the current extension study was that although parent study blinding was maintained, both patients and site personnel knew that all participants were receiving bapineuzumab. There may have been imbalances in analyses between the pbo $\rightarrow$ bapi and 
bapi $\rightarrow$ bapi groups due to differences in study completion rates between treatment groups in the parent studies. The sample sizes for the biomarker substudies were small.

\section{Conclusion}

Infusion of bapineuzumab 0.5 or $1.0 \mathrm{mg} / \mathrm{kg}$ every 13 weeks for up to 3 years was generally well tolerated, with a safety and tolerability profile that was similar to the previous parent studies. The long-term safety and tolerability profile was generally similar in $A P O E \& 4$ carriers and noncarriers, except for the higher incidence of ARIA-E in carriers. The incidence of extension-onset ARIA-E in both noncarriers and carriers was higher in those who initially received bapineuzumab during the extension study than in those who had received bapineuzumab since the start of the parent study. This finding confirmed the decline in the incidence of ARIA-E over time with continued bapineuzumab exposure, which was observed during the parent double-blind studies and was consistent with a reduced risk of ARIA-E after longer exposure. There were no other noteworthy differences in safety between those groups and analysis of TEAEs did not detect any new safety signals for bapineuzumab.

When patients from the parent studies received bapineuzumab 0.5 or $1.0 \mathrm{mg} / \mathrm{kg}$ IV in the longterm extension Study 351, similar deterioration of cognition and function occurred in the pbo $\rightarrow$ bapi and bapi $\rightarrow$ bapi groups. The results were similar for $A P O E \& 4$ carriers and noncarriers. There were no statistically significant differences between those who received bapineuzumab in both the parent and extension studies and those who first received bapineuzumab in the extension study. Overall, these results confirm that bapineuzumab at these doses did not slow the clinical or pathophysiologic progression of $\mathrm{AD}$.

\section{ACKNOWLEDGMENTS}

The authors are most grateful to the study patients for their contributions and the investigational staff for the medical care. The authors acknowledge Bradford Challis (Janssen Research \& Development, LLC) for assistance in preparation and editorial support of the manuscript. The study was funded by Janssen Alzheimer Immunotherapy Research \& Development LLC, and Pfizer Inc.

Authors' disclosures available online (https:// www.j-alz.com/manuscript-disclosures/17-1157r1).

\section{SUPPLEMENTARY MATERIAL}

The supplementary material is available in the electronic version of this article: http://dx.doi.org/ $10.3233 / \mathrm{jad}-171157$.

\section{REFERENCES}

[1] Bard F, Cannon C, Barbour R, Burke RL, Games D, Grajeda H, Guido T, Hu K, Huang J, Johnson-Wood K, Khan K, Kholodenko D, Lee M, Lieberburg I, Motter R, Nguyen M, Soriano F, Vasquez N, Weiss K, Welch B, Seubert P, Schenk D, Yednock T (2000) Peripherally administered antibodies against amyloid beta-peptide enter the central nervous system and reduce pathology in a mouse model of Alzheimer disease. Nat Med 6, 916-919.

[2] Miles LA, Crespi GA, Doughty L, Parker MW (2013) Bapineuzumab captures the N-terminus of the Alzheimer's disease amyloid-beta peptide in a helical conformation. Sci Rep 3, 1302.

[3] Salloway S, Sperling R, Fox NC, Blennow K, Klunk W, Raskind M, Sabbagh M, Honig LS, Porsteinsson AP, Ferris S, Reichert M, Ketter N, Nejadnik B, Guenzler V, Miloslavsky M, Wang D, Lu Y, Lull J, Tudor IC, Liu E, Grundman M, Yuen E, Black R, Brashear HR, Bapineuzumab, Clinical Trial I (2014) Two phase 3 trials of bapineuzumab in mild-to-moderate Alzheimer's disease. $N$ Engl J Med 370, 322-333.

[4] Vandenberghe R, Rinne JO, Boada M, Katayama S, Scheltens P, Vellas B, Tuchman M, Gass A, Fiebach JB, Hill D, Lobello K, Li D, McRae T, Lucas P, Evans I, Booth K, Luscan G, Wyman BT, Hua L, Yang L, Brashear HR, Black RS, Bapineuzumab 3000 and 3001 Clinical Study Investigators (2016) Bapineuzumab for mild to moderate Alzheimer's disease in two global, randomized, phase 3 trials. Alzheimers Res Ther 8, 18 .

[5] Abushouk AI, Elmaraezy A, Aglan A, Salama R, Fouda S, Fouda R, AlSafadi AM (2017) Bapineuzumab for mild to moderate Alzheimer's disease: A meta-analysis of randomized controlled trials. BMC Neurol 17, 66 .

[6] Blennow K, De Meyer G, Hansson O, Minthon L, Wallin A, Zetterberg H, Lewczuk P, Vanderstichele H, Vanmechelen E, Kornhuber J, Wiltfang J, Group KN-S, Heuser I, Maier W, Luckhaus C, Ruther E, Hull M, Jahn H, Gertz HJ, Frolich L, Hampel H, Pernetzki R (2009) Evolution of Abeta42 and Abeta40 levels and Abeta42/Abeta40 ratio in plasma during progression of Alzheimer's disease: A multicenter assessment. J Nutr Health Aging 13, 205-208.

[7] Blennow K, Hampel H (2003) CSF markers for incipient Alzheimer's disease. Lancet Neurol 2, 605-613.

[8] Blennow K, Zetterberg H, Rinne JO, Salloway S, Wei J, Black R, Grundman M, Liu E, Investigators AAB (2012) Effect of immunotherapy with bapineuzumab on cerebrospinal fluid biomarker levels in patients with mild to moderate Alzheimer disease. Arch Neurol 69, 1002-1010.

[9] Liu E, Schmidt ME, Margolin R, Sperling R, Koeppe R, Mason NS, Klunk WE, Mathis CA, Salloway S, Fox NC, Hill DL, Les AS, Collins P, Gregg KM, Di J, Lu Y, Tudor IC, Wyman BT, Booth K, Broome S, Yuen E, Grundman M, Brashear HR, Bapineuzumab 301 and 302 Clinical Trial Investigators (2015) Amyloid-beta 11C-PiB-PET imaging results from 2 randomized bapineuzumab phase $3 \mathrm{AD}$ trials. Neurology 85, 692-700. 
[10] Rinne JO, Brooks DJ, Rossor MN, Fox NC, Bullock R, Klunk WE, Mathis CA, Blennow K, Barakos J, Okello AA, Rodriguez Martinez de Liano S, Liu E, Koller M, Gregg KM, Schenk D, Black R, Grundman M (2010) 11CPiB PET assessment of change in fibrillar amyloid-beta load in patients with Alzheimer's disease treated with bapineuzumab: A phase 2, double-blind, placebo-controlled, ascending-dose study. Lancet Neurol 9, 363-372.

[11] Sperling R, Salloway S, Brooks DJ, Tampieri D, Barakos J, Fox NC, Raskind M, Sabbagh M, Honig LS, Porsteinsson AP, Lieberburg I, Arrighi HM, Morris KA, Lu Y, Liu E, Gregg KM, Brashear HR, Kinney GG, Black R, Grundman M (2012) Amyloid-related imaging abnormalities in patients with Alzheimer's disease treated with bapineuzumab: A retrospective analysis. Lancet Neurol 11, 241-249.

[12] Ketter N, Brashear HR, Bogert J, Di J, Miaux Y, Gass A, Purcell DD, Barkhof F, Arrighi HM (2017) Central review of amyloid-related imaging abnormalities in two phase III clinical trials of bapineuzumab in mild-to-moderate Alzheimer's disease patients. J Alzheimers Dis 57, 557-573.

[13] Ivanoiu A, Pariente J, Booth K, Lobello K, Luscan G, Hua L, Lucas P, Styren S, Yang L, Li D, Black RS, Brashear HR, McRae T (2016) Long-term safety and tolerability of bapineuzumab in patients with Alzheimer's disease in two phase 3 extension studies. Alzheimers Res Ther 8, 24.

[14] Sperling R, Salloway S, van Dyck C, Brashear H, Ketter N, Banerjee K, Morris K, Arrighi M, Lobello K, Guenzler V (2013) Clinical correlates of amyloid-related imaging abnormalities (ARIA) in phase 3 studies of bapineuzumab in Alzheimer's disease (AD) (S44.002). Neurology 80, S44.002-S044.002.

[15] Schott JM, Bartlett JW, Fox NC, Barnes J, Alzheimer's Disease Neuroimaging Initiative (2010) Increased brain atrophy rates in cognitively normal older adults with low cerebrospinal fluid Abeta1-42. Ann Neurol 68, 825-834.

[16] Chan D, Janssen JC, Whitwell JL, Watt HC, Jenkins R, Frost C, Rossor MN, Fox NC (2003) Change in rates of cerebral atrophy over time in early-onset Alzheimer's disease: Longitudinal MRI study. Lancet 362, 1121-1122.

[17] Sperling RA, Jack CR Jr, Aisen PS (2011) Testing the right target and right drug at the right stage. Sci Transl Med 3, $111 \mathrm{~cm} 133$.
[18] Doody RS, Thomas RG, Farlow M, Iwatsubo T, Vellas B, Joffe S, Kieburtz K, Raman R, Sun X, Aisen PS, Siemers E, Liu-Seifert H, Mohs R, Alzheimer's Disease Cooperative Study Steering Committee; Solanezumab Study Group (2014) Phase 3 trials of solanezumab for mild-to-moderate Alzheimer's disease. N Engl J Med 370, 311-321.

[19] Panza F, Solfrizzi V, Imbimbo BP, Giannini M, Santamato A, Seripa D, Logroscino G (2014) Efficacy and safety studies of gantenerumab in patients with Alzheimer's disease. Expert Rev Neurother 14, 973-986.

[20] Sevigny J, Chiao P, Bussiere T, Weinreb PH, Williams L, Maier M, Dunstan R, Salloway S, Chen T, Ling Y, O'Gorman J, Qian F, Arastu M, Li M, Chollate S, Brennan MS, Quintero-Monzon O, Scannevin RH, Arnold HM, Engber T, Rhodes K, Ferrero J, Hang Y, Mikulskis A, Grimm J, Hock C, Nitsch RM, Sandrock A (2016) The antibody aducanumab reduces Abeta plaques in Alzheimer's disease. Nature 537, 50-56.

[21] Relkin NR, Thomas RG, Rissman RA, Brewer JB, Rafii MS, van Dyck CH, Jack CR, Sano M, Knopman DS, Raman R, Szabo P, Gelmont DM, Fritsch S, Aisen PS, Alzheimer's Disease Cooperative Study (2017) A phase 3 trial of IV immunoglobulin for Alzheimer disease. Neurology 88, 1768-1775.

[22] de la Torre JC (2014) Phase 3 trials of solanezumab and bapineuzumab for Alzheimer's disease. $N$ Engl J Med 370, 1459-1460.

[23] Doody RS, Farlow M, Aisen PS, Alzheimer's Disease Cooperative Study Data Analysis and Publication Committee (2014) Phase 3 trials of solanezumab and bapineuzumab for Alzheimer's disease. N Engl J Med 370, 1460.

[24] Laske C (2014) Phase 3 trials of solanezumab and bapineuzumab for Alzheimer's disease. $N$ Engl J Med 370, 1459.

[25] Salloway S, Sperling R, Brashear HR (2014) Phase 3 trials of solanezumab and bapineuzumab for Alzheimer's disease. N Engl J Med 370, 1460.

[26] Sperling RA, Rentz DM, Johnson KA, Karlawish J, Donohue M, Salmon DP, Aisen P (2014) The A4 study: Stopping AD before symptoms begin? Sci Transl Med 6, 228fs213. 